\title{
Methanol as a diagnostic tool of interstellar clouds
}

\section{Model calculations and application to molecular clouds}

\author{
S. Leurini ${ }^{1}$, P. Schilke ${ }^{1}$, K. M. Menten ${ }^{1}$, D. R. Flower ${ }^{2}$, J. T. Pottage ${ }^{2}$, and L.-H. Xu ${ }^{3}$ \\ 1 Max-Planck-Institut für Radioastronomie, Auf Dem Hügel 69, 53121, Bonn, Germany \\ e-mail: [sleurini; schilke;kmenten] @mpifr-bonn.mpg.de \\ 2 Physics Department, University of Durham, DH1 3LE, UK \\ e-mail: [david.flower; j.t.pottage]@durham.ac.uk \\ 3 Physical Sciences Department, University of New Brunswick, Saint John, NB, E2L 4L5, Canada \\ e-mail: xuli@unb.ca
}

Received 9 January 2004 / Accepted 26 March 2004

\begin{abstract}
We present a detailed analysis of the diagnostic properties of methanol, $\left(\mathrm{CH}_{3} \mathrm{OH}\right)$, in dense molecular clouds, made possible by the availability of new $\left(\mathrm{CH}_{3} \mathrm{OH}-\mathrm{He}\right)$ collisional rate coefficients. Using a spherical Large Velocity Gradient (LVG) model, the dependence on kinetic temperature and spatial density of various millimeter and submillimeter line bands is investigated over a range of physical parameters typical of high- and low-mass star-forming regions. We find $\mathrm{CH}_{3} \mathrm{OH}$ to be a good tracer of high-density environments and sensitive to the kinetic temperature. Using our LVG model, we have also developed an innovative technique to handle the problem of deriving physical parameters from observed multi-line spectra of a molecule, based on the simultaneous fit of all the lines with a synthetic spectrum, finding the best physical parameters using numerical methods.
\end{abstract}

Key words. ISM: molecules - ISM: clouds - molecular processes

\section{Introduction}

Kinetic temperature and density are fundamental parameters for our understanding of the interstellar medium (ISM). However, since the main gas-phase molecular component, $\mathrm{H}_{2}$, is not directly observable under "normal" conditions, one has to rely on other molecules to trace the physical state of an interstellar cloud. Usually, symmetric rotors such as $\mathrm{NH}_{3}$ are used to probe a cloud's kinetic temperature (Walmsley \& Ungerechts 1983; Danby et al. 1988), while linear molecules, e.g. CS, are used to determine its density (e.g., Snell et al. 1984; Beuther et al. 2002). However, different spatial distributions of the tracers ("chemistry") often complicate the picture, (see, e.g., Tafalla et al. 2002) as they often trace physically different and spatially non-coexisting gas components. It is thus desirable to trace all relevant physical parameters with a single molecule.

Promising candidates exist among slightly asymmetric rotors, which have properties qualifying them as tracers for physical conditions. Since they are almost symmetric, they share a strong sensitivity to kinetic temperature with symmetric molecules, but they also allow determinations of spatial density (for a detailed discussion of $\mathrm{H}_{2} \mathrm{CO}$, see Mundy et al. 1987; Mangum \& Wootten 1993).
Methanol, $\mathrm{CH}_{3} \mathrm{OH}$, a slightly asymmetric rotor, is a proven tracer of high-density environments (e.g. Menten et al. 1988), but up to now an extremely poor knowledge of the $\mathrm{CH}_{3} \mathrm{OH}$ collisional rates and of their propensity rules has prevented realistic systematic studies exploiting methanol's full potential as an interstellar tracer. Recently, this situation has changed with the calculation of collisional rate coefficients by Pottage et al. (2001, 2002), which we have integrated in a "standard" Large Velocity Gradient program aimed at modelling methanol excitation. Here, we present an application of our model calculations to observations of clouds without IR radiation, while extensive modelling of a variety of evolved star-forming regions as well as Class I methanol masers will be discussed in accompanying papers. In Sect. 2 we discuss a detailed analysis of the tracing properties of $\mathrm{CH}_{3} \mathrm{OH}$ in dense molecular clouds; in Sect. 3 we present a new approach to derive physical parameters from $\mathrm{CH}_{3} \mathrm{OH}$ spectral lines.

\section{Excitation of $\mathrm{CH}_{3} \mathrm{OH}$}

Due to the threefold symmetry of its hindering potential, $\mathrm{CH}_{3} \mathrm{OH}$ occurs as $A$ - and $E$-symmetry states. The $E$-type, $-J \leq k \leq J$, is vibrationally doubly degenerate: the $E_{1}, k \geq 0$, states are degenerated with $E_{2}, k \leq 0$. In the $A$-type, $0 \leq K \leq J$, 


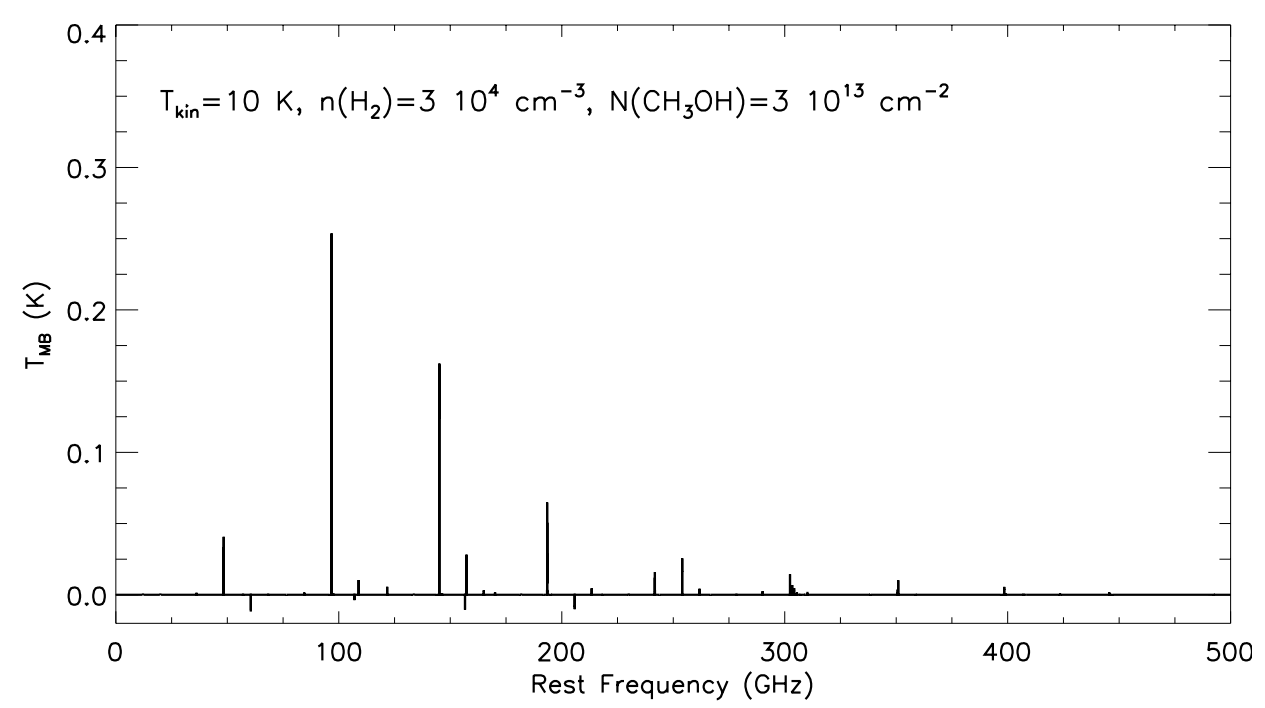

(a)

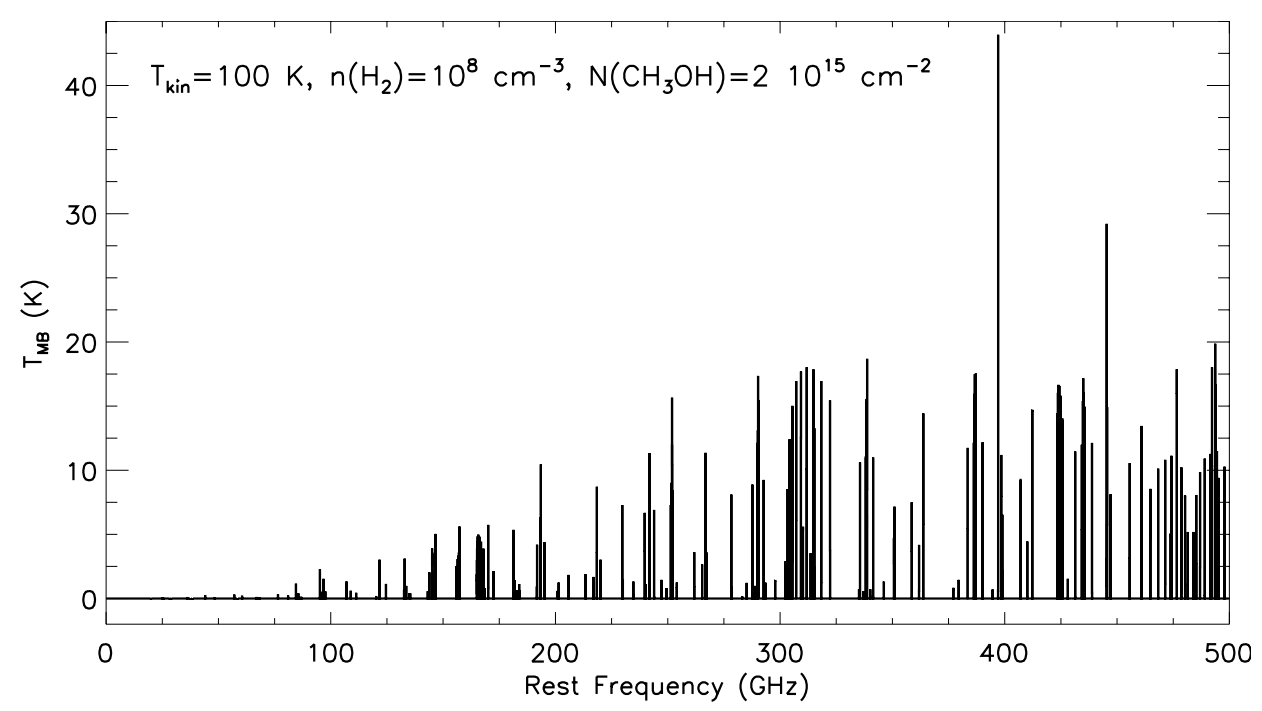

(b)

Fig. 1. Synthetic $\mathrm{CH}_{3} \mathrm{OH}$ spectra, (source size $=20^{\prime \prime}$, telescope diameter $=30 \mathrm{~m}$ ), up to $(J, k)=9$ for the torsional ground state, calculated using our LVG model.

for $K \geq 0$ there are doublets split by asymmetry which are labelled $A^{+}$and $A^{-}$(Lees \& Baker 1968). For radiative transport purposes, its $A$ - and $E$-type symmetry states can be considered as two different molecules, since interconversion can happen only through proton exchange reactions, which happen on timescales so long that the excitation is unaffected. Transitions between $A^{+}$and $A^{-}$levels occur.

$\mathrm{CH}_{3} \mathrm{OH}$ is ubiquitous and associated with different regimes of star formation, from quiescent, cold $(T \sim 10 \mathrm{~K})$, dark clouds, where its abundance relative to $\mathrm{H}_{2}$ is only $\sim 10^{-9}$ (Friberg et al. 1988), to "hot core" sources near high-mass (proto) stellar objects, where $\left[\mathrm{CH}_{3} \mathrm{OH} / \mathrm{H}_{2}\right]$ values $\sim 10^{-7}-10^{-6}$ are observed (Menten et al. 1986, 1988).

A practical advantage is that, because of its complex energy level structure, it has groups of several transitions close together in wavelength space throughout the millimeter and submillimeter region that can be observed simultaneously, thus minimizing relative calibration uncertainties, and a multitude of its transitions is observable with ground-based telescopes.

Methanol has in the past been used as a probe of spatial density in dense molecular clouds, (cf. Friberg et al. 1988; Walmsley et al. 1988; Menten et al. 1988; Kalenskii et al. 1997; Bachiller et al. 1998); however, uncertainties in the derived densities are expected since these authors modelled the $\mathrm{CH}_{3} \mathrm{OH}$ excitation using collisional rates based upon the experiments by Lees \& Haque (1974) for $\mathrm{CH}_{3} \mathrm{OH}$ with $\mathrm{He}$, for low states of $\mathrm{CH}_{3} \mathrm{OH}-\mathrm{E}$ only.

The $\mathrm{CH}_{3} \mathrm{OH}$ spectrum up to $500 \mathrm{GHz}$ is shown in Fig. 1 for two different sets of physical parameters typical for the ISM calculated with our LVG program. Rest frequencies are from the Cologne Database for Molecular Spectroscopy, (http: //www . cdms. de, Müller et al. 2001), which includes new measurements by Xu \& Lovas (1997). 


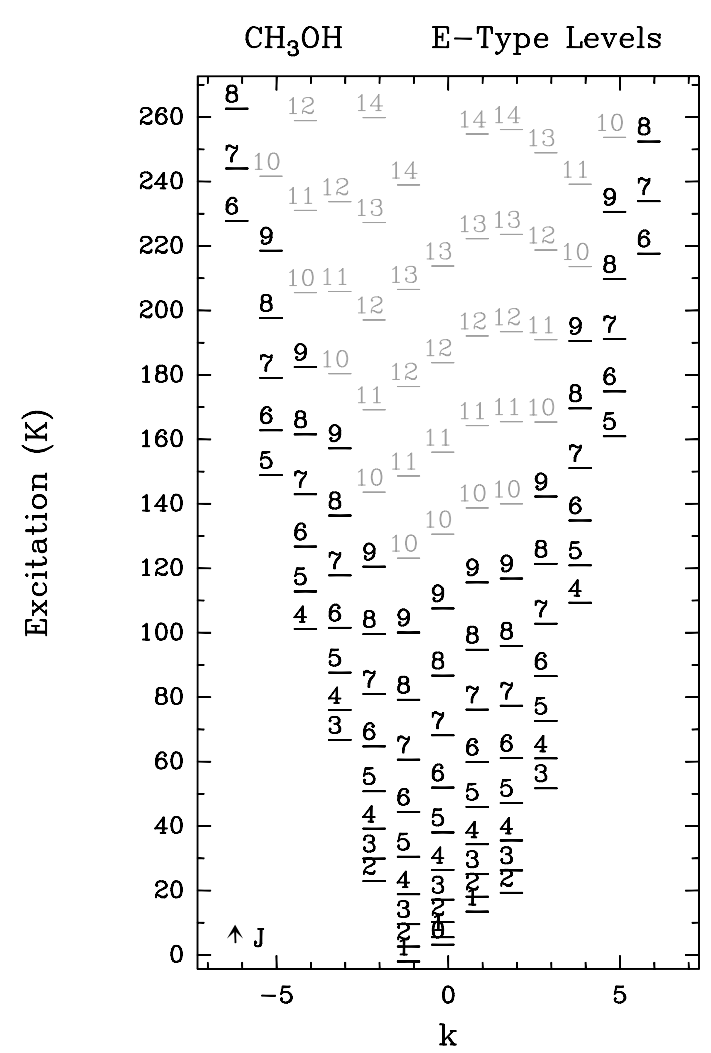

(a)

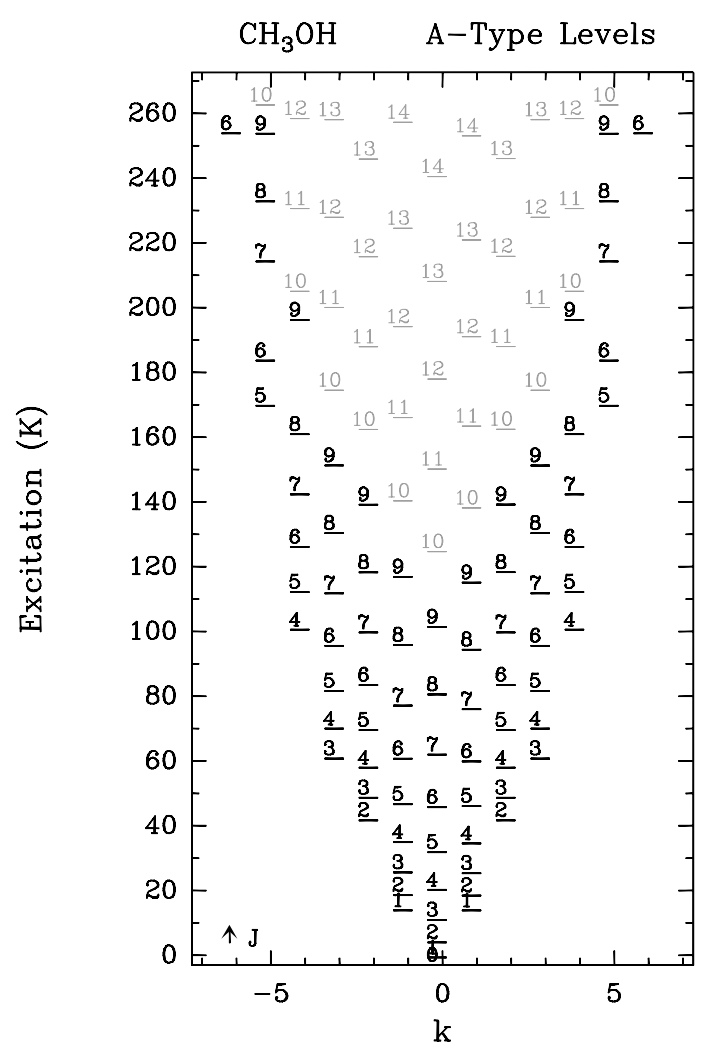

(b)

Fig. 2. Energy levels for the $E$ - and $A$-symmetry states of $\mathrm{CH}_{3} \mathrm{OH}$ in the torsional ground state with an excitation energy of less than $260 \mathrm{~K}$ and $J<9$, (black), $9<J<14$, (grey). Levels for $\mathrm{CH}_{3} \mathrm{OH}-A^{+}$and $\mathrm{CH}_{3} \mathrm{OH}-A^{-}$have been included in the same diagram: $k<0$ indicates levels from $\mathrm{CH}_{3} \mathrm{OH}-A^{-}, k>0$ levels from $\mathrm{CH}_{3} \mathrm{OH}-A^{+}$.

\section{1. $\mathrm{CH}_{3} \mathrm{OH}-\mathrm{He}$ collisional rates}

Only recently (see Pottage et al. 2001, 2002), rate coefficients for collisions of methanol with helium, for both $\mathrm{CH}_{3} \mathrm{OH}-\mathrm{A}$ and $\mathrm{CH}_{3} \mathrm{OH}-E$, have been computed for levels up to $\left(J, k^{1}\right)=9$ at kinetic temperatures up to $200 \mathrm{~K}$. Calculations of rate coefficients for collisions with para- $\mathrm{H}_{2}$ have been recently carried out and comparisons of our results with this new dataset will be analysed in a forthcoming paper. With this new set of collisional rates, we have carried out statistical equilibrium calculations on both $A$ - and $E$-types for the torsional ground state, using the Large Velocity Gradient (LVG) method with spherical geometry in the derivation of de Jong et al. (1975). Since the new calculations on collisional rates have been computed only for the torsional ground state, we limit our study to those sources in which the only external radiation field is the cosmic background radiation, in order to avoid any effect due to infrared pumping. We estimate differences in the interaction potentials between $\mathrm{He}$ and $\mathrm{H}_{2}$ to be likely more important than those due to their different reduced masses and did not scale the $\mathrm{CH}_{3} \mathrm{OH}-\mathrm{He}$ collisional rates by any factor related to the masses.

1 The projection of the angular momentum quantum number $k$ runs from $-J$ to $+J$ for $E$-type $\mathrm{CH}_{3} \mathrm{OH}$. For $A$-type $\mathrm{CH}_{3} \mathrm{OH}$ a capital $K$ is used, with $0<K \leq J$. When referring in one expression to levels from both species, lowercase $k$ is used.
Our calculations are extended to the first 100 levels for each state, for which the collisional rates from Pottage et al. (2001, 2002) are available; the coverage in energy is thus incomplete above $100 \mathrm{~K}$, (see Figs. 2a,b), and does not allow a reliable estimation of the partition function at high temperatures. We compared our LTE values for the partition function with the one obtained with a more complete set of levels, $(J \leq 26, k \leq 14)$, for which energies are calculated using global fit parameters reported in Xu \& Hougen (1995), and we expect no significant uncertainties in our results up to $50 \mathrm{~K}$, where the deviation from the most accurate value of $Q$ is close to $10 \%$. Since our sample of sources is not expected to show temperatures higher than $50 \mathrm{~K}$, the inaccuracy in the partition function should not affect our calculations. All allowed radiative transitions for the levels up to $(J, k)=9$ have been included in our model, even the weak $\Delta k \geq 2$ lines. Line strengths are from Mekhtiev et al. (1999).

\subsection{First test - dark clouds}

Pottage et al. (2001) have compared their results with the ones from Lees \& Haque (1974) and tested the accuracy of their collisional rates by modelling the $2_{0} \rightarrow 3_{-1} E$ transition at $12.18 \mathrm{GHz}$. They find a generally good agreement with the measurements of Lees \& Haque (1974), with exceptions, for example, in the $\Delta k=3 m$ transitions, (where $m$ is an integer). We extended the tests to other transitions observed in dark clouds, where any external radiation field but the cosmic 


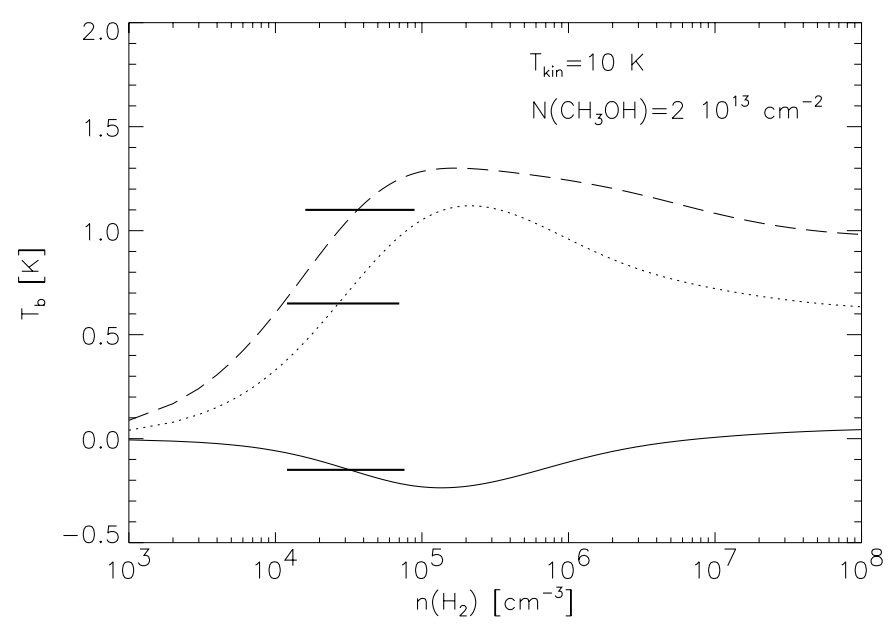

Fig. 3. Results of statistical equilibrium calculations. We show the $2_{0} \rightarrow 3_{-1} E$, (full line), the $2_{-1} \rightarrow 1_{-1} E$, (dotted line) and the $2_{0} \rightarrow 1_{0} A$, (long dashed line), line temperatures as a function of $\mathrm{H}_{2}$ density. Superimposed on the plot, the observed values towards L134N from Walmsley et al. (1988), and Friberg et al. (1988) are indicated by horizontal lines.

background is negligible and level populations are expected to be governed by collisions. Figure 3 shows the model results for the $2_{0} \rightarrow 3_{-1} E$ line at $12.18 \mathrm{GHz}$ and the $2_{-1} \rightarrow 1_{-1} E$ and $2_{0} \rightarrow 1_{0} A$ transitions near $96 \mathrm{GHz}$; typical dark clouds parameters, $\left(n\left(\mathrm{H}_{2}\right)=10^{3}-10^{8} \mathrm{~cm}^{-3}, T=10 \mathrm{~K}\right)$, were assumed in order to model the lines and compare the predictions with the observations from Walmsley et al. (1988) and Friberg et al. (1988). L134N has been selected as test source, since detailed multi-transition studies on it exist (see Swade 1989; Dickens et al. 2000). Dickens et al. (2000) find an average density along several lines of sight of $\sim 2 \times 10^{4} \mathrm{~cm}^{-3}$, from $\mathrm{HC}_{3} \mathrm{~N}, \mathrm{~N}_{2} \mathrm{H}^{+}$ and $\mathrm{CS}$, while $\mathrm{NH}_{3}$ observations give a kinetic temperature of $10 \mathrm{~K}$. Assuming an $A / E$ abundance ratio equal to 1 , they derive a $\mathrm{CH}_{3} \mathrm{OH}$ column density in the range $10^{13}-10^{14} \mathrm{~cm}^{-2}$. Following Walmsley et al. (1988), the $2_{0} \rightarrow 3_{-1} E$ data towards L134N have been corrected by a factor 0.5 due to an offset position of $3^{\prime}$ relative to the $2_{0} \rightarrow 1_{0} A$ peak from Friberg et al. (1988). Our results are shown in Fig. 3: all three lines indicate a $\mathrm{H}_{2}$ density in the range $\sim 3-4 \times 10^{4} \mathrm{~cm}^{-3}$ that is in good agreement with the "average" density toward various lines of sight in this source $\left(\sim 2 \times 10^{4} \mathrm{~cm}^{-3}\right)$ determined by Dickens et al. (2000) from a variety of molecules.

Our results are generally in good agreement with old models of methanol excitation and suggest that estimates of spatial density based on Lees \& Haque (1974) collisional rates are generally reliable. However our tests regard only the $12.18 \mathrm{GHz}$ line and the quartet at $96 \mathrm{GHz}$ and we did not carry out a systematic comparison of the dependence on spatial density and kinetic temperature of the $\mathrm{CH}_{3} \mathrm{OH}$ transitions computed with the collisional rates from Pottage et al. (2001, 2002) and with the ones based on the experiment by Lees \& Haque (1974).

\section{3. $\mathrm{CH}_{3} \mathrm{OH}$ as a probe of interstellar physical conditions}

The richness of the $\mathrm{CH}_{3} \mathrm{OH}$ spectrum prevents us from a systematic study of all its transitions: taking into account only the energy levels with $(J, k) \leq 9$, the $E$ state alone has more than 300 lines, (see Figs. 1a,b). Therefore, we restricted our study to only some bands observable from ground based telescope and not showing any maser action: the $2_{k} \rightarrow 1_{k}-A$ and -E-type lines near $96 \mathrm{GHz}$, the $5_{k} \rightarrow 4_{k}-A$ and $-E$ lines near $241 \mathrm{GHz}$, the $7_{k} \rightarrow 6_{k}-A$ and $-E$ lines near $338 \mathrm{GHz}$. Even if promising candidates to trace kinetic temperature are in the submillimeter bands, with current collisional rates, we cannot reliably analyse higher transitions because the level set is incomplete above $100 \mathrm{~K}$, (see Figs. 2a,b).

As a first step, we analysed line ratios from transitions close together in frequency space, in order to find diagnostic tools of kinetic temperature and density which are not affected by beam-size differences, pointing and absolute calibration uncertainties. A slight asymmetry in the abundances of the $A$ and $E$ subspecies, due to a difference in their ground state energies, has been suggested and indeed observed (see Friberg et al. 1988; Menten et al. 1988). Since their relative abundance can vary from 0.69 in dark clouds, (Friberg et al. 1988), to 2 in OMC-2, (Menten et al. 1988), comparisons between $\mathrm{CH}_{3} \mathrm{OH}-E$ and $-A$ lines are not taken into account to avoid any uncertainties in deriving physical parameters due to an inaccurate knowledge of the $A / E$ ratio.

From the $\mathrm{CH}_{3} \mathrm{OH}-\mathrm{E}$ selected transitions, we found several ratios to be calibration-independent tracers of density: the $T_{R}\left(2_{1} \rightarrow 1_{1}\right) / T_{R}\left(2_{-1} \rightarrow 1_{-1}\right)$ and the $T_{R}\left(2_{0} \rightarrow 1_{0}\right) / T_{R}\left(2_{-1} \rightarrow\right.$ $\left.1_{-1}\right)$ in the $96 \mathrm{GHz}$ band; the $T_{R}\left(5_{0} \rightarrow 4_{0}\right) / T_{R}\left(5_{-1} \rightarrow 4_{-1}\right)$, the $T_{R}\left(5_{1} \rightarrow 4_{1}\right) / T_{R}\left(5_{-1} \rightarrow 4_{-1}\right)$, the $T_{R}\left(5_{2} \rightarrow 4_{2}\right) / T_{R}\left(5_{-1} \rightarrow 4_{-1}\right)$, and the $T_{R}\left(5_{-2} \rightarrow 4_{-2}\right) / T_{R}\left(5_{-1} \rightarrow 4_{-1}\right)$ in the $241 \mathrm{GHz}$ band. They all also show a dependence on temperature in the range $5-15 \mathrm{~K}$ that suggest their use as temperature tracer in dark clouds. However, no line ratios in the $1 \mathrm{~mm}$ and the $3 \mathrm{~mm}$ bands show a pure dependence on the kinetic temperature, while for several ratios in the $1 \mathrm{~mm}$ band we found a strong sensitivity to both density and temperature.

The submillimeter band we analysed, at $338 \mathrm{GHz}$, shows a behaviour very similar to the $1 \mathrm{~mm}$ series, with several line ratios being sensitive to both kinetic temperature and spatial density. However, some line ratios can be used as calibrationindependent tracers of density, (e.g. the $T_{R}\left(7_{0} \rightarrow 6_{0}\right) / T_{R}\left(7_{-1} \rightarrow\right.$ $\left.6_{-1}\right)$ and the $\left.T_{R}\left(7_{1} \rightarrow 6_{1}\right) / T_{R}\left(7_{-1} \rightarrow 6_{-1}\right)\right)$. A pure dependence on kinetic temperature is found in line ratios with $|k| \geq 3$, but mainly at low density $\left(n\left(\mathrm{H}_{2} \leq 10^{5} \mathrm{~cm}^{-3}\right)\right.$ where these lines are very weak (see Figs. 6a,b). However, given the sensitivity to both kinetic temperature and spatial density of several line ratios in the $5_{k} \rightarrow 4_{k}$ and $7 k \rightarrow 6_{k}$ bands and the pure dependence on density of others, the simultaneous observations of several lines from these series allows determining both the physical parameters.

Figures 4-6 show LVG model predictions for line ratios in logarithmic scale as function of density and temperature at different column densities. The submillimeter bands are studied only at high column densities $\left(10^{14}-10^{16} \mathrm{~cm}^{-2} /\left(\mathrm{km} \mathrm{s}^{-1}\right)\right)$ 


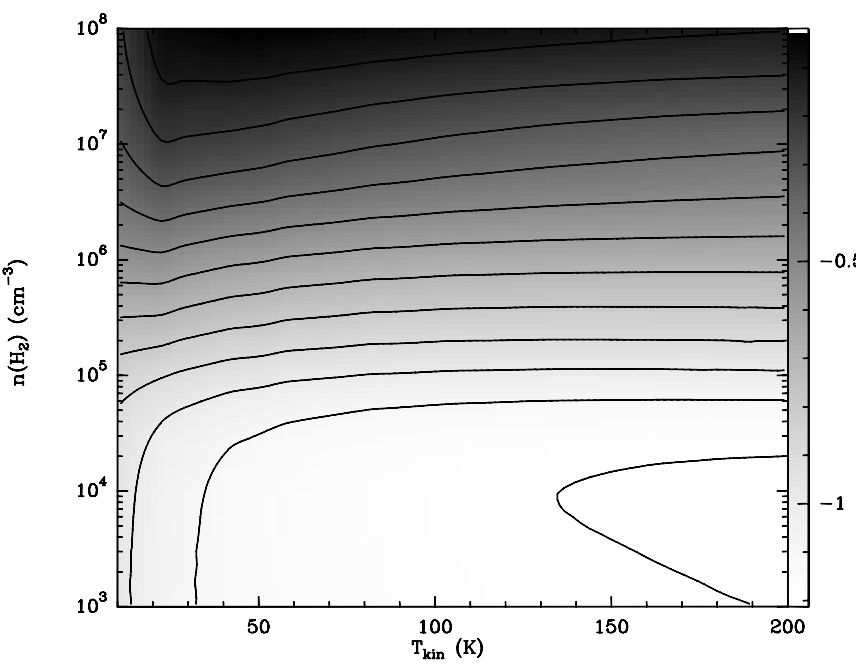

(a)

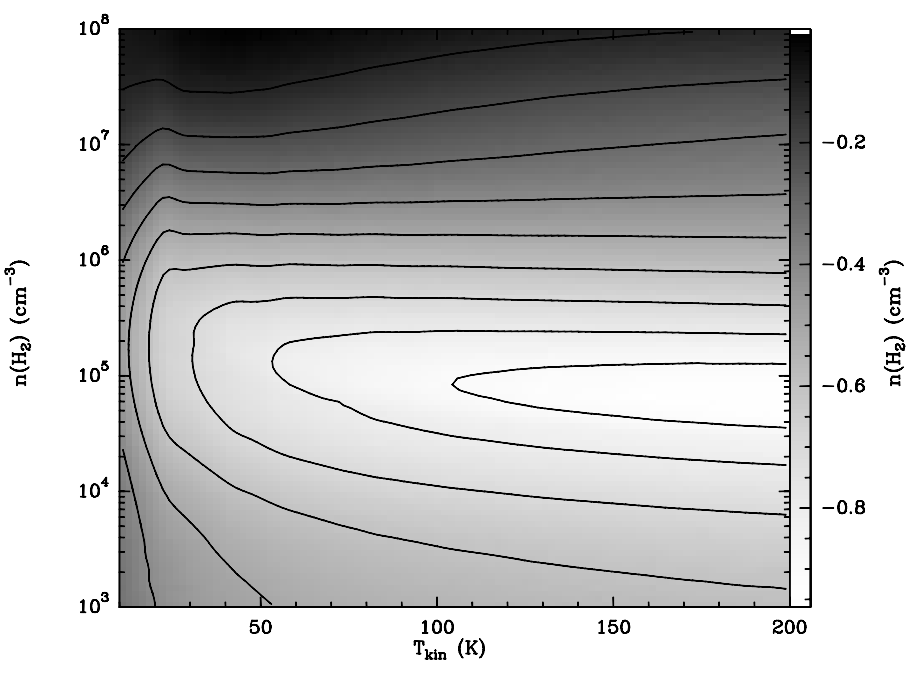

(c)

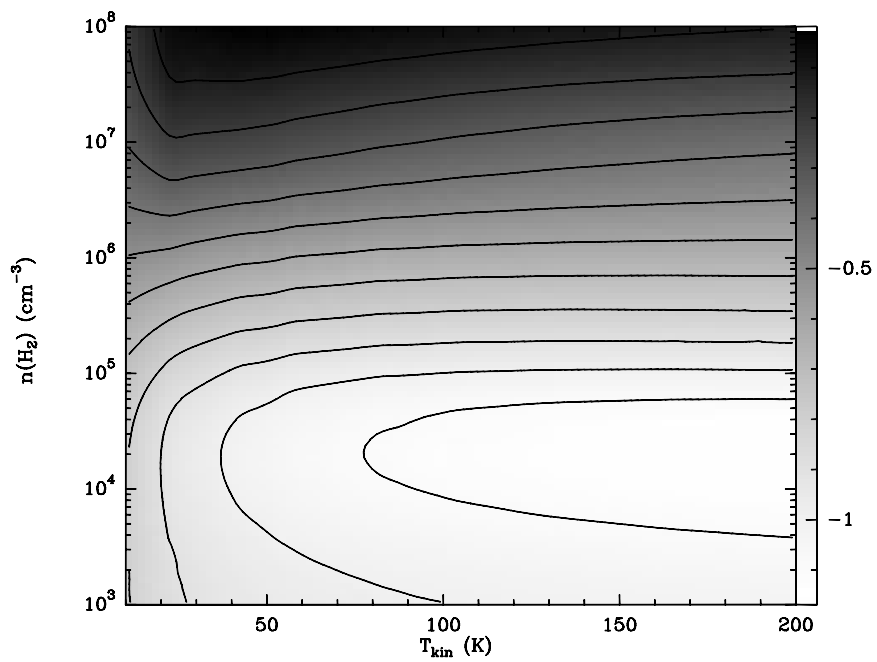

(b)

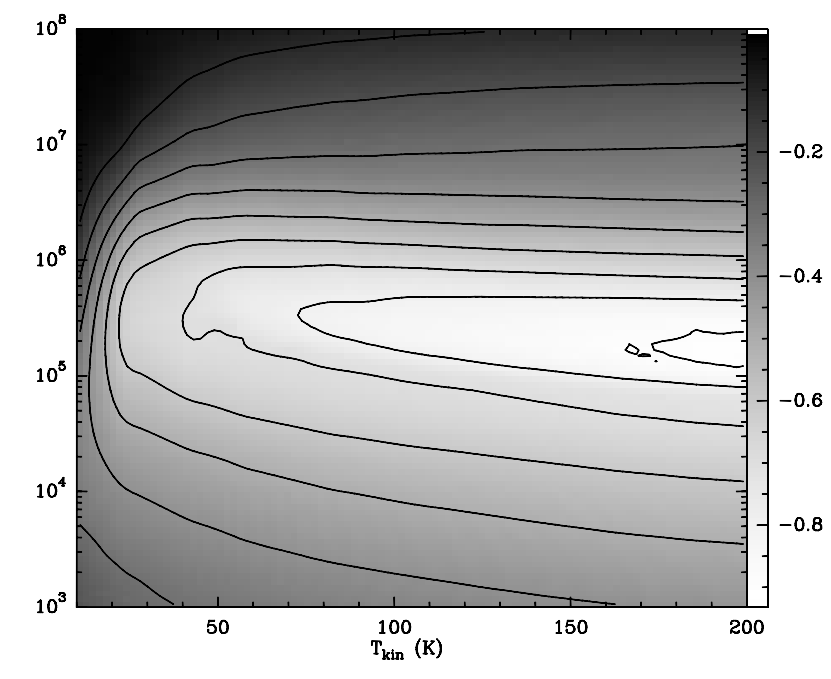

(d)

Fig. 4. Results of statistical equilibrium calculations for $\mathrm{CH}_{3} \mathrm{OH}-E$. The $T_{R}\left(2_{0} \rightarrow 1_{0}\right) / T_{R}\left(2_{-1} \rightarrow 1_{-1}\right)$ line ratio is shown in logarithmic scale, as function of $\mathrm{H}_{2}$ density and temperature at $N\left(\mathrm{CH}_{3} \mathrm{OH}\right) / \Delta v=10^{13}$, a) $N\left(\mathrm{CH}_{3} \mathrm{OH}\right) / \Delta v=10^{14}$, b) $N\left(\mathrm{CH}_{3} \mathrm{OH}\right) / \Delta v=10^{15}$, c) and $N(\mathrm{CH} 3 \mathrm{OH}) / \Delta v=$ $10^{16} \mathrm{~cm}^{-2} /\left(\mathrm{km} \mathrm{s}^{-1}\right)$. d) The black contours range from -1.5 to 0 by steps of 0.1 . The whole $T-n$ plane is accessible to observations.

where their brightness temperatures become significant. Our results are summarized in the following guidelines:

- line ratios in the $2_{k} \rightarrow 1_{k}$ and the $5_{k} \rightarrow 4_{k}$ bands at 96 and $241 \mathrm{GHz}$ are tracers of densities higher than $10^{5} \mathrm{~cm}^{-3}$, with a weak dependence on temperatures up to $15 \mathrm{~K}$;

- line ratios in the $5_{k} \rightarrow 4_{k}$ at $241 \mathrm{GHz}$ and $7_{k} \rightarrow 6_{k}$ at $338 \mathrm{GHz}$ are sensitive to kinetic temperature and spatial density, with some examples of pure density tracers;

- information on kinetic temperature is recovered when lines with an excitation temperature close to the expected kinetic temperature of the source are observed.

The main result we found is that, for all the analysed millimeter bands, ratios between lines in the same band are strongly sensitive to density, with a weaker dependence on temperature.
This is explained by the high probability of the $a$-type ${ }^{2}$ radiative transitions to occur, $\left(A_{i j} \propto \mu^{2}, \mu_{a}=0.896, \mu_{b}=1.412\right.$, Sastry et al. 1981). Even if $\mathrm{CH}_{3} \mathrm{OH}$ is only slightly asymmetric, $\left(\kappa=-0.98\right.$ for $\mathrm{CH}_{3} \mathrm{OH}$, while $\kappa=-1$ for a prolate symmetric rotor, where $\kappa$ is the Ray's asymmetry parameter, Ray 1932; Townes \& Schawlow 1996), the $\Delta k \neq 0$ radiative lines are strongly allowed and not mainly dependent on the collisions as the structural similarity of asymmetric molecules with symmetric rotors would suggest. However, in the submillimeter regime, several transitions show a strong dependence on the kinetic temperature in the range up to $100-150 \mathrm{~K}$. Nevertheless, since several line ratios in the $5_{k} \rightarrow 4_{k}$ and in the $7_{k} \rightarrow 6_{k}$ band are strongly dependent on kinetic temperature and

${ }^{2}$ Using standard asymmetric rotor nomenclature, $a$-type transitions have $\Delta k=0$, while $b$-type transitions have $\Delta k+/-1$. 


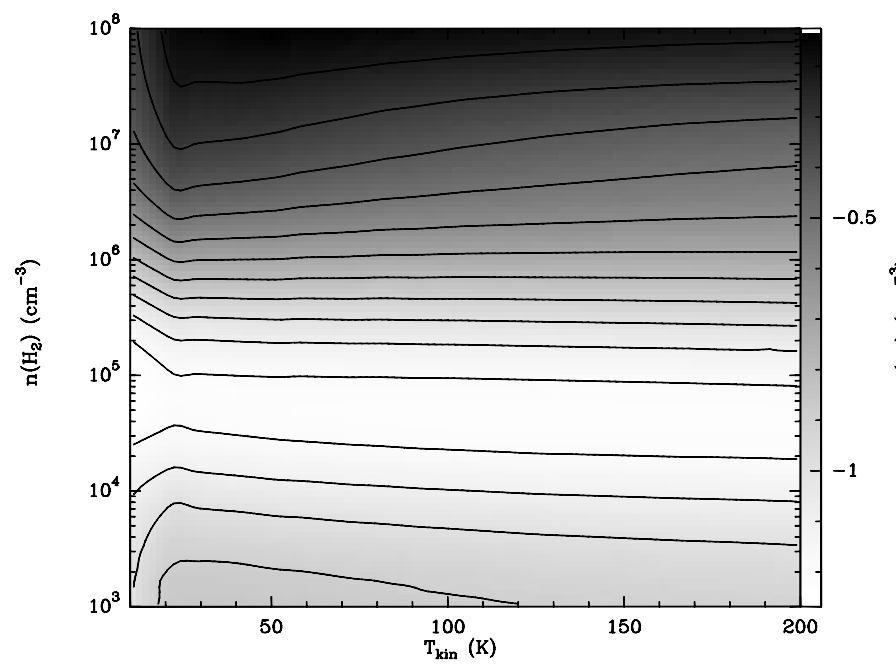

(a)

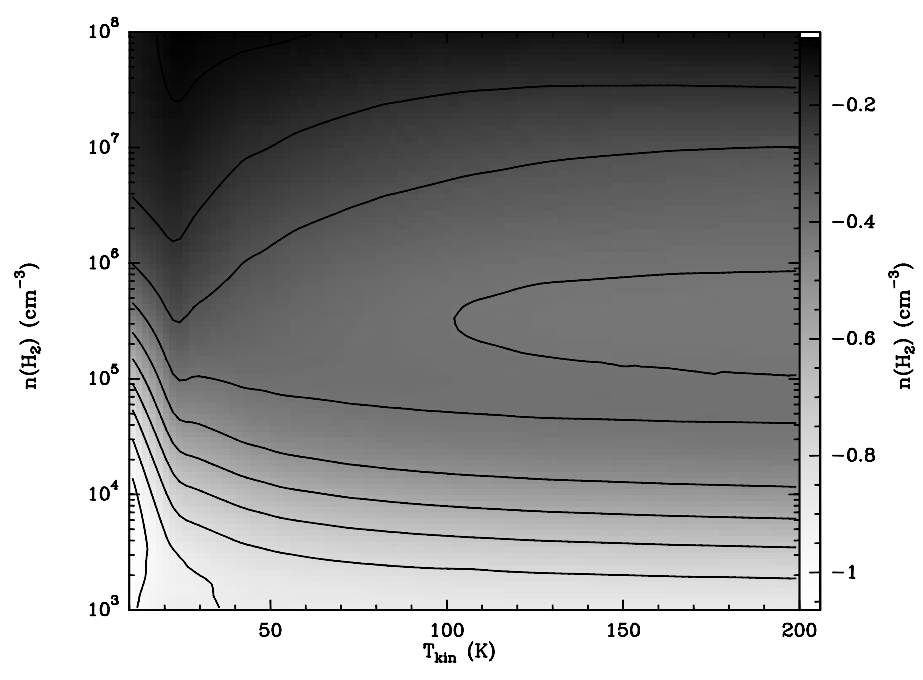

(c)

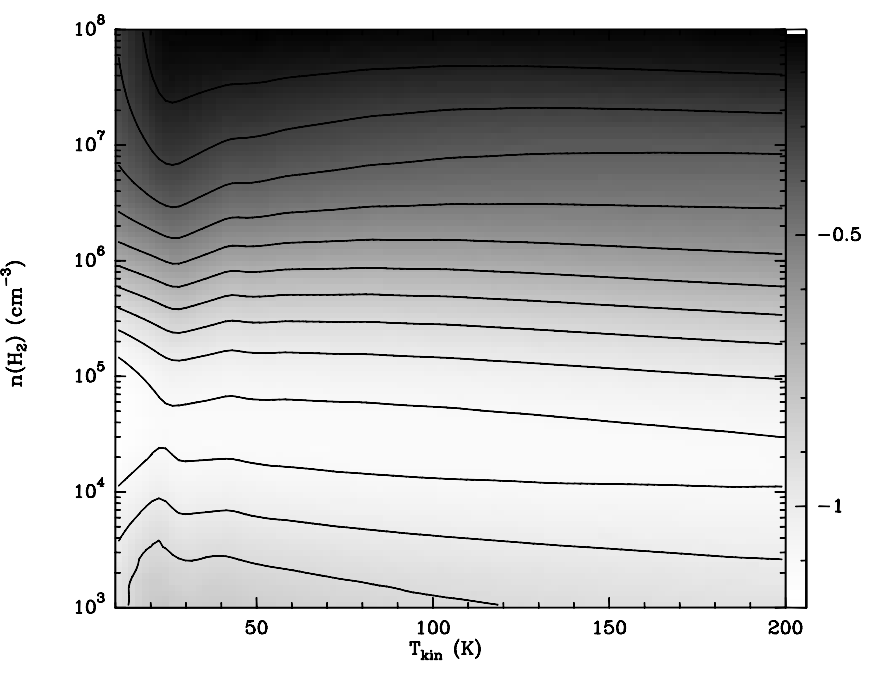

(b)

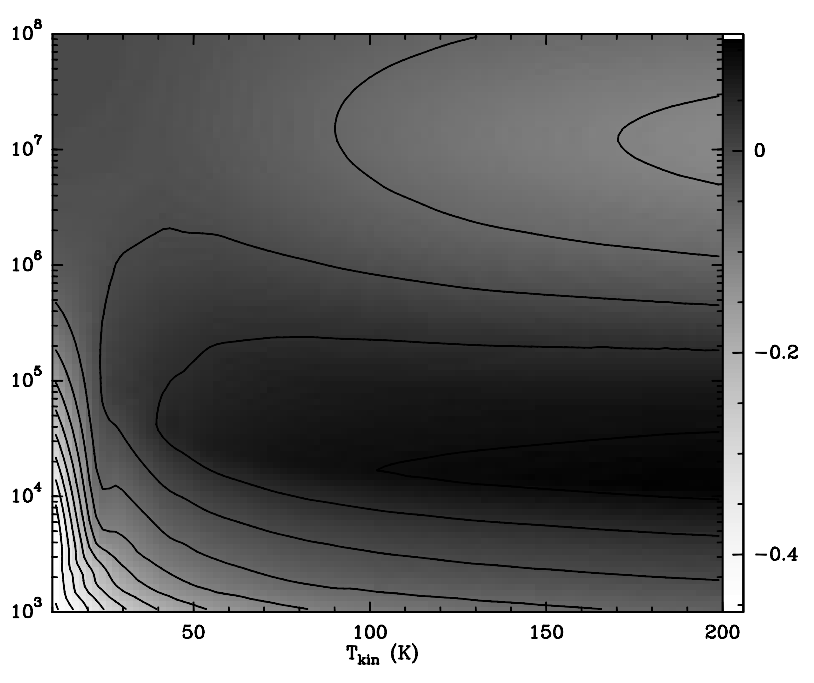

(d)

Fig. 5. Results of statistical equilibrium calculations for $\mathrm{CH}_{3} \mathrm{OH}-E$. The $T_{R}\left(5_{0} \rightarrow 4_{0}\right) / T_{R}\left(5_{-1} \rightarrow 4_{-1}\right)$ line ratio in logarithmic scale is shown as function of $\mathrm{H}_{2}$ density and temperature at $N\left(\mathrm{CH}_{3} \mathrm{OH}\right) / \Delta v=10^{13}$, a) $N\left(\mathrm{CH}_{3} \mathrm{OH}\right) / \Delta v=10^{14}$, b) $N\left(\mathrm{CH}_{3} \mathrm{OH}\right) / \Delta v=10^{15}$, c) and $N\left(\mathrm{CH}{ }_{3} \mathrm{OH}\right) / \Delta v=$ $10^{16} \mathrm{~cm}^{-2} /\left(\mathrm{km} \mathrm{s}^{-1}\right)$. d) The black contours range from -1.5 to 0 by steps of 0.1 in a), b) and c); from -0.5 to 0.1 by steps of 0.05 in d). The whole $T-n$ plane is accessible to observations.

spatial density, both the parameters can be derived when a rich $\mathrm{CH}_{3} \mathrm{OH}$ spectrum is available, with lines in different frequency and excitation ranges. Line ratios from the $5_{k} \rightarrow 4_{k}$ band with the $7_{k} \rightarrow 6_{k}$ series show a dependence on temperature up to $50-80 \mathrm{~K}$, at high density, $n\left(\mathrm{H}_{2}\right) \geq 10^{6} \mathrm{~cm}^{-3}$, (see Figs. 7a,b), since they have different excitation energies.

\section{Analysis technique}

The traditional approach for deriving physical parameters such as kinetic temperature and spatial density from an observed spectrum involves "by-hand" Gaussian fitting of the lines with multiple components and $\chi^{2}$ analysis comparing the measured quantities with statistical equilibrium calculations (see Olmi et al. 1993; Mangum \& Wootten 1993). Better results are obtained if the analysed quantities are ratios between lines close together in frequency space and observed simultaneously with the same receiver, because uncertainties due to absolute calibration and to the beam filling factor are minimized. The main drawback is the loss of information on the column density. On the other hand, when working with line intensities, uncertainties due to absolute calibration and to the beam filling factor play a major role in making the determination of physical parameters less reliable. In general, this kind of analysis also ignores upper limits on lines too weak to be detected, because of the non-physical fitting procedure. For complex sources, the technique also requires decomposing line profiles, a tedious and, for strongly blended lines, very unreliable process. When applied to large multi-line datasets, like line surveys of a source 


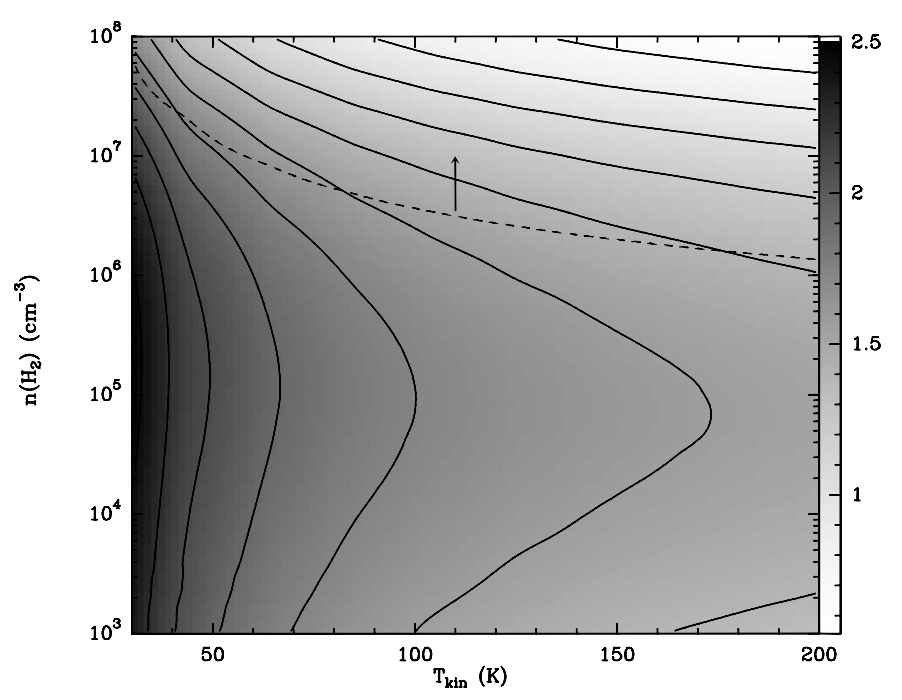

(a)

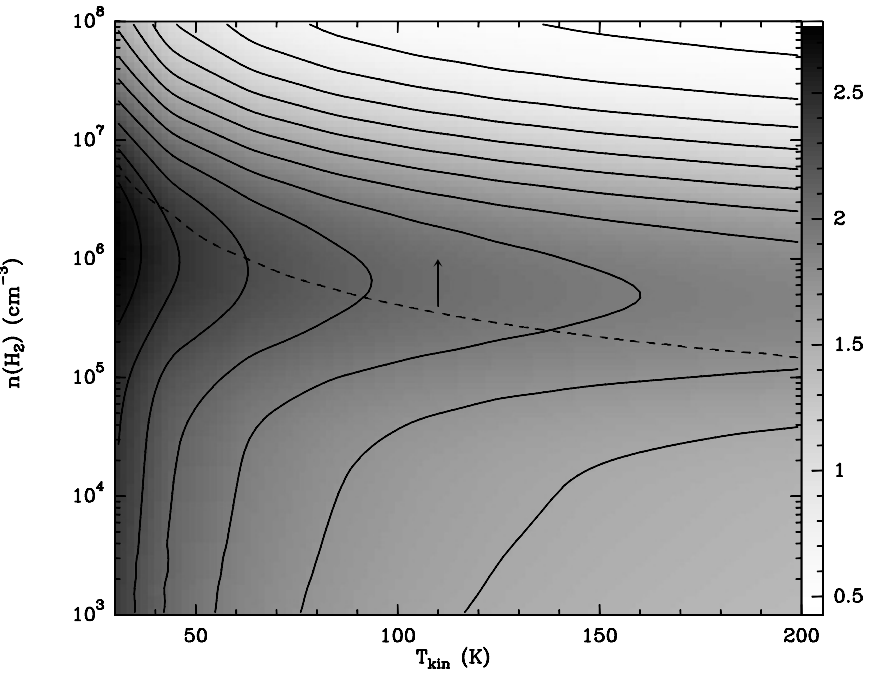

(b)

Fig. 6. Results of statistical equilibrium calculations for $\mathrm{CH}_{3} \mathrm{OH}-E$. a) The $T_{R}\left(7_{-3} \rightarrow 6_{-3}\right) / T_{R}\left(7_{5} \rightarrow 6_{5}\right)$ line ratio in logarithmic scale, as function of $\mathrm{H}_{2}$ density and temperature at $N\left(\mathrm{CH}_{3} \mathrm{OH}\right) / \Delta v=10^{15}$, and b) at $10^{16} \mathrm{~cm}^{-2} /\left(\mathrm{km} \mathrm{s}^{-1}\right)$. The black contours range from 0 to 2.5 by steps of 0.2 . The black dashed line corresponds to a $7_{5} \rightarrow 6_{5}$ line intensity of $0.01 \mathrm{~K}$; a black arrow shows how the $T_{R}\left(7_{5} \rightarrow 6_{5}\right)$ increases with spatial density; the plane below the black dashed line is therefore inaccessible to observations.

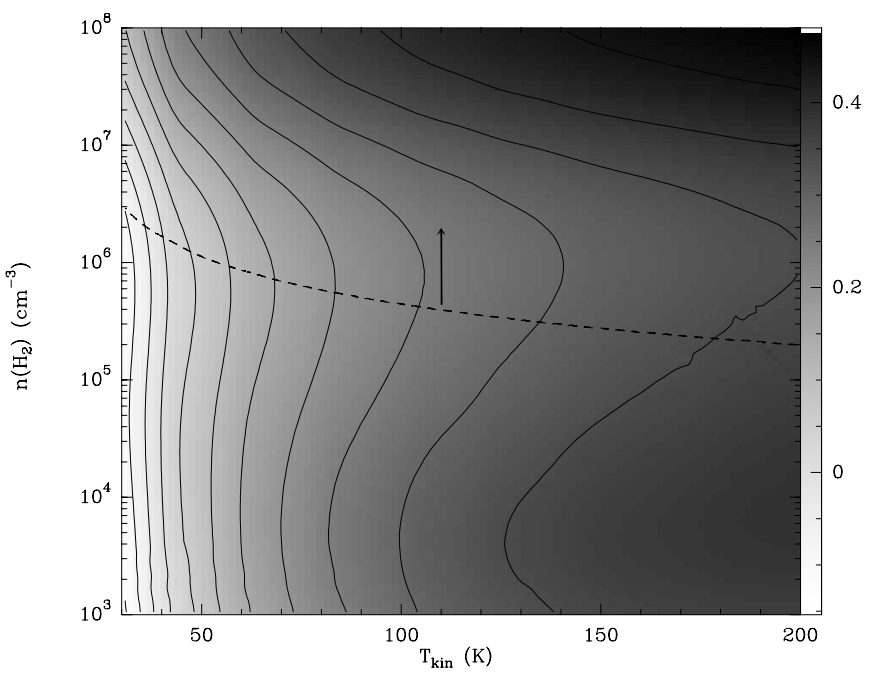

(a)

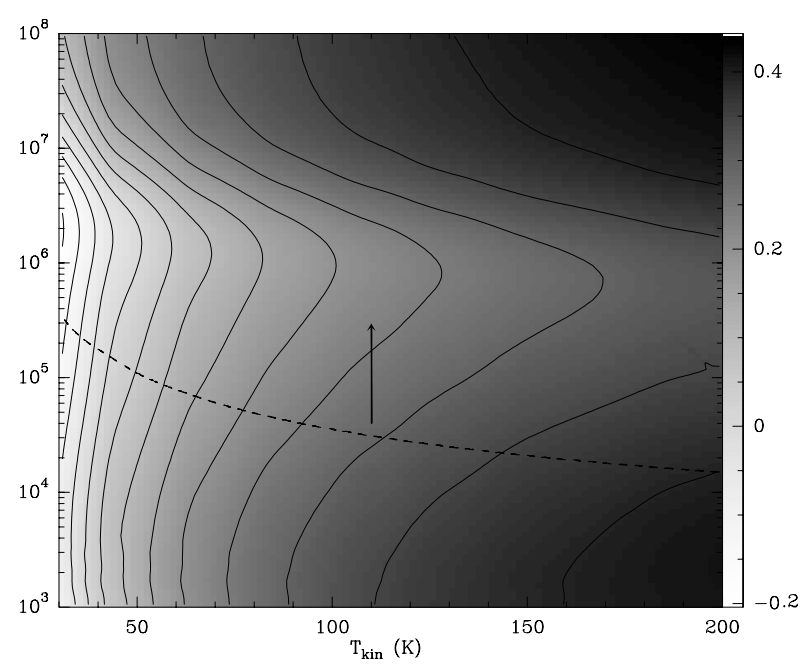

(b)

Fig. 7. Results of statistical equilibrium calculations for $\mathrm{CH}_{3} \mathrm{OH}-E$. a) The $T_{R}\left(5_{-3} \rightarrow 4_{-3}\right) / T_{R}\left(7_{-4} \rightarrow 6_{-4}\right)$ line ratio in logarithmic scale, as function of $\mathrm{H}_{2}$ density and temperature at $N\left(\mathrm{CH}_{3} \mathrm{OH}\right) / \Delta v 10^{15}$, and b) at $10^{16} \mathrm{~cm}^{-2} /\left(\mathrm{km} \mathrm{s}^{-1}\right)$. The black contours range from -0.3 to 1 by steps of 0.05 . The black dashed contour corresponds to a $7_{5} \rightarrow 6_{5}$ line intensity of $0.01 \mathrm{~K}$; a black arrow shows how the $T_{R}\left(7_{5} \rightarrow 6_{5}\right)$ increases with spatial density; the plane below the black dashed line is therefore inaccessible to observations.

or systematic multi-frequency studies of a molecule, by-hand fitting is impratical. With sensitive receivers available nowadays and in view of the next generation of instruments, which will provide copious amounts of data in a short time, new methods of data analysing and modelling are required.

An innovative technique to handle the problem, proposed by Schilke et al. (1999) and recently improved by Comito et al. (2004), is based on the simultaneous fit of all the lines in a spectrum with a synthetic spectrum computed under Local Thermodynamic Equilibrium (LTE) conditions. Here we propose an extension to this technique using the LVG approximation: this development is necessary and fully justifies the computing-time involved in such an analysis because we expect prominent departures from LTE in the sources of our sample and in many other astrophysical scenarios. To allow for complexity in the physical conditions along a line of sight, our model handles multiple components, which are assumed not to interact, i.e. the intensities simply add up. The free parameters for each component are kinetic temperature, molecular hydrogen density, source size and column density. To take into account the possibly different abundance between $A$ and $E$ states, $\mathrm{CH}_{3} \mathrm{OH}-\mathrm{A}$ and $\mathrm{CH}_{3} \mathrm{OH}-\mathrm{E}$ column densities are treated as two independent free parameters. Line width, $\Delta v$, and LSR velocity, $v_{\mathrm{LSR}}$, are fixed parameters and assumed to be the same for all the lines in each component. The fitting is done by minimizing the $\chi^{2}$ between the data and the model spectrum using 
the Levenberg-Marquardt method from Press et al. (1992). The $\chi^{2}$ analysis is however not trivial, given the high number of fitted channels. We assume the degrees of freedom to be given by $v_{\mathrm{d}}=N_{\mathrm{o}}-N_{\mathrm{f}}$, where $N_{\mathrm{o}}$, the number of observed quantities, is here the number of all the channels in which $\mathrm{CH}_{3} \mathrm{OH}$ transitions fall, even if not detected, plus the observed velocity and line width of each components. $N_{\mathrm{f}}$ is the number of free parameters. Frequencies for the line identification are from the Cologne Database for Molecular Spectroscopy. In the current implementation, 100000 lines can be fitted simultaneously and data from different telescopes can be combined easily.

All the levels for which the collisional rates from Pottage et al. (2002) are available are used for the calculations; however, at low temperatures $\left(T_{\text {kin }} \leq 30 \mathrm{~K}\right)$ the program runs into numerical problems due to the depopulation of the most excited levels in this regime. Therefore, only a subset of transitions is used in this range.

We assume the following representation of the spectrum:

$T_{v}=\sum_{c} \eta\left(\theta^{c}\right)\left[\tilde{J}\left(T_{\mathrm{ex}_{v}}^{c}\right)-J\left(T_{\mathrm{bg}}\right)\right]\left(1-\mathrm{e}^{-\tilde{\tau}_{v}^{c}}\right)$

with

$\tilde{\tau}_{v}^{c}=\sum_{l} \tau_{l}^{\mathrm{c}}=\sum_{l} \frac{c^{3}}{8 \pi v_{l}^{3}} A_{l} \frac{N_{\mathrm{CH}_{3} \mathrm{OH}}^{c}}{\Delta v^{c}}\left(n_{\text {low }}^{c} \frac{g_{\mathrm{up}}}{g_{\text {low }}}-n_{\mathrm{up}}^{c}\right) \phi^{l, c}$

and

$\tilde{J}\left(T_{\mathrm{ex}_{\nu}}^{c}\right)=\frac{\sum_{l} J\left(T_{\mathrm{ex}_{l}}^{c}\right) \tau_{l}^{c}}{\sum_{l} \tau_{l}^{c}}$

where $T_{\mathrm{ex}}$ and $\tau$ are given by the LVG results, $\eta$ is the beam filling factor and $\phi$ the line profile. The sums are computed on both $A$ and $E$ states; the indices $l$ and $c$ correspond to lines and spatial components. In Eq. (2), $n_{\text {low }}^{c}$ and $n_{\text {up }}^{c}$ are the fractional populations of the lower and upper levels for the transition $l$ in the component $c$ and depend on the set of levels used for the calculations.

An implicit assumption, when solving the radiative transport equation with the escape probability method, is that different lines do not overlap locally. This is not true for molecules with a complex spectrum, especially for $\mathrm{CH}_{3} \mathrm{OH}$, whose $A$ and $E$ transitions are often very close in the frequency space. Following Cesaroni \& Walmsley (1991), we define an average optical depth and brightness temperature by means of the previous expressions Eqs. (2) and (3) when lines have a frequency separation

$v_{i}-v_{j} \leq \Delta v_{i}+\Delta v_{j}$

Equation (3) is equivalent to Eq. (13) of Cesaroni \& Walmsley (1991) in units of temperature. In the optically thin limit, Eq. (3) is equal to the traditional approach of convolving a line with several Gaussians. On the other hand, it better describes situations in which both optically thin and optically thick lines are present: photons emitted from the optically thin transition are locally absorbed by the optically thick emission. The intensities of the lines do not simply add up like in the optically thin limit, but the intensity at the overlapping frequencies is mainly described by the optically thick emission. In the simplest case of one component and two transitions, both optically thin, Eq. (1) can be approximated by

$$
\begin{aligned}
T_{v}= & \eta\left(\theta^{c}\right)\left[J\left(T_{\mathrm{ex}_{1}}^{c}\right)-J\left(T_{\mathrm{bg}}\right)\right] \tau_{1}^{c} \\
& +\eta\left(\theta^{c}\right)\left[J\left(T_{\mathrm{ex}_{2}}^{c}\right)-J\left(T_{\mathrm{bg}}\right)\right] \tau_{2}^{c}
\end{aligned}
$$

while when one of the two lines, e.g. line 1 , is optically thick

$$
\begin{aligned}
T_{v}= & \frac{1}{\tau_{1}^{c}+\tau_{2}^{c}} \eta\left(\theta^{c}\right)\left[J\left(T_{\mathrm{ex}_{1}}^{c}\right)-J\left(T_{\mathrm{bg}}\right)\right] \tau_{1}^{c} \\
& +\frac{1}{\tau_{1}^{c}+\tau_{2}^{c}} \eta\left(\theta^{c}\right)\left[J\left(T_{\mathrm{ex}_{2}}^{c}\right)-J\left(T_{\mathrm{bg}}\right)\right] \tau_{2}^{c} \\
\simeq & \eta\left(\theta^{c}\right)\left[J\left(T_{\mathrm{ex}_{1}}^{c}\right)-J\left(T_{\mathrm{bg}}\right)\right] .
\end{aligned}
$$

\subsection{Tests on the convergence criterion}

Although the simultaneous fit of a spectrum has been demonstrated to be a powerful technique to derive physical parameters (Comito et al. 2004), it does imply several approximations. The assumption behind the analysis is that the $\mathrm{CH}_{3} \mathrm{OH}$ emission can be reasonably well approximated by a small number of non-interacting components, thus ignoring any alignments between them. This assumption is fully justified when no information on the morphology is available, but it fails in well reproducing the observed spectra if the components are related, leading, for example, to overestimate line intensities when self-absorption happens. The LVG approach adds other uncertainties to the obtained results, since it assumes only one set of physical parameters for each component used for fitting the source and implicitly neglects any local and nonlocal overlap between the lines; a more detailed model, including density and temperature distributions within each component, would be desirable. However, fitting large datasets with a Monte Carlo synthetic spectrum and minimizing all the free parameters would involve an enormous amount of computing time and it is practically impossible to be applied to a sample of sources. In fact, most (if not all) published Monte Carlo fits used manual fitting of the parameters, with no control over reading a globally best fit, or assessment to the uniqueness of the fit (e.g., Hogerheijde \& van der Tak 2000). Moreover, since several spectral windows can be fitted simultaneously, calibration uncertainties still render the determination of physical parameters less reliable. Another drawback is the uniqueness of the $\chi^{2}$ minimization, which is not a priori guaranteed. Indeed the minimum found occasionally depends on the input parameters. A better control on the fit results is assured when plausible input parameters are used. Particularly important is the information on the source size, which helps in solving the degeneracy between source size, temperature and column density for certain parameter ranges. In the worst situation, no information at all is available in the literature on the source to be modelled and the number of free parameters is $5 \times N_{\mathrm{c}}$, where $N_{\mathrm{c}}$ the number of components. To investigate whether the found solution is local or global, an analysis of the $\chi^{2}$ distribution as function of the different free parameters in an interval reasonably close to the minimum is desirable. 
Table 1. $\mathrm{CH}_{3} \mathrm{OH}$ model results.

\begin{tabular}{|c|c|c|c|c|c|c|c|}
\hline & $\begin{array}{c}v \\
\left(\mathrm{~km} \mathrm{~s}^{-1}\right)\end{array}$ & $\begin{array}{l}T_{K} \\
(\mathrm{~K})\end{array}$ & $\begin{array}{l}n\left(\mathrm{H}_{2}\right) \\
\left(\mathrm{cm}^{-3}\right)\end{array}$ & $\begin{array}{c}N\left(\mathrm{CH}_{3} \mathrm{OH}-A\right) \\
\left(\mathrm{cm}^{-2}\right)\end{array}$ & $\begin{array}{c}N\left(\mathrm{CH}_{3} \mathrm{OH}-E\right) \\
\left(\mathrm{cm}^{-2}\right)\end{array}$ & $\begin{array}{c}\text { source size } \\
\left({ }^{\prime \prime}\right)\end{array}$ & $\overline{\chi_{v_{\mathrm{d}}}^{2}}$ \\
\hline \multicolumn{8}{|c|}{ best fit } \\
\hline G19.30P1 & & & & & & & 3 \\
\hline Core & 3.0 & 44 & $10^{6}$ & $7.5 \times 10^{15}$ & $3 \times 10^{15}$ & 5 & \\
\hline Outflow & 10.0 & 24 & $1.8 \times 10^{5}$ & $210^{15}$ & $2 \times 10^{15}$ & 12 & \\
\hline \multicolumn{8}{|c|}{$1 \sigma$ fit range } \\
\hline \multicolumn{8}{|l|}{ G19.30P1 } \\
\hline Core & & $25-320$ & $1.2 \times 10^{5}-3 \times 10^{6}$ & $3.6 \times 10^{15}-1.6 \times 10^{16}$ & $2 \times 10^{15}-5 \times 10^{15}$ & & \\
\hline Outflow & & $18-61$ & $4.5 \times 10^{4}-3 \times 10^{5}$ & $\begin{array}{l}1.4 \times 10^{15}-3 \times 10^{15} \\
\text { best fit }\end{array}$ & $1 \times 10^{15}-2.5 \times 10^{15}$ & & \\
\hline \multirow[t]{2}{*}{ G79.3P1 } & & & & & & & 0.2 \\
\hline & 2.0 & 17 & $2.510^{5}$ & $7.9 \times 10^{13}$ & $5.7 \times 10^{13}$ & 32 & \\
\hline \multicolumn{8}{|c|}{$1 \sigma$ fit range } \\
\hline \multicolumn{8}{|l|}{ G79.3P1 } \\
\hline & & $12-52$ & $4 \times 10^{4}-7 \times 10^{5}$ & $5 \times 10^{13}-1.3 \times 10^{14}$ & $3.6 \times 10^{13}-8.1 \times 10^{13}$ & & \\
\hline
\end{tabular}

However, one should be aware of the limits in determining the physical parameters of a source which are intrinsic to this technique. Unless lines with $\tau \ll 1$ and $\tau \gg 1$ are available, solving the degeneracy between source size and column density in the optically thin limit, $\left(T(v)=\sum \eta\left[J\left(T_{\mathrm{ex}}^{c}\right)-J\left(T_{\mathrm{bg}}\right)\right] \tau\right)$, where the information on the temperature is recovered when several optically thin lines are available, or between temperature and source size, in the optically thick case, $(T(v)=$ $\left.\sum \eta\left[J\left(T_{\mathrm{ex}}^{c}\right)-J\left(T_{\mathrm{bg}}\right)\right]\right)$, is impossible. Plotting the optical depth for each fitted line gives a better idea on which of the obtained parameters is more affected by uncertainties. Also, fitting $\mathrm{CH}_{3} \mathrm{OH}$ spectra assures a reliable determination of kinetic temperature only when many lines are fitted, thus covering different excitation ranges, and/or when millimeter and submillimeter data are available, (see Sect. 2.3).

Observations of the $2_{k} \rightarrow 1_{k}-A$ and $-E$ lines near $96 \mathrm{GHz}$, the $J_{0} \rightarrow J_{-1}-E$ lines at $157 \mathrm{GHz}$, the $5_{k} \rightarrow 4_{k}-A$ and $-E$ near $241 \mathrm{GHz}$ were obtained towards a sample of more than 60 sources during a systematic program on $\mathrm{CH}_{3} \mathrm{OH}$ in highmass star forming regions at the IRAM $30 \mathrm{~m}$ telescope during summer 2002-winter 2003. Details on the observations and analysis of the data are given in a forthcoming paper.

We here discuss the application of our method to two sources of our sample, G19.30P1 and G79.3P1. They both belong to a population of infrared-dark clouds, (hereafter IRDCs), identified by Egan et al. (1998) using the Galactic plane survey from the SPIRIT III telescope aboard the Midcourse Space Experiment, MSX. LVG calculations on $\mathrm{H}_{2} \mathrm{CO}$, (Carey et al. 1998), give an average value of $T \leq 20 \mathrm{~K}, n\left(\mathrm{H}_{2}\right) \geq 10^{5} \mathrm{~cm}^{-3}$ and $N\left(\mathrm{H}_{2} \mathrm{CO}\right) \sim 10^{13}-10^{14} \mathrm{~cm}^{-2}$ for IRDCs. Pillai et al. (2004) derive a kinetic temperature of $17 \mathrm{~K}$ for G19.30P1 and $15 \mathrm{~K}$ for $\mathrm{G} 79.3 \mathrm{P} 1$ from $\mathrm{NH}_{3}(1,1),(2,2)$ data; typical values for $\mathrm{H}_{2}$ column densities are $10^{22}-10^{23} \mathrm{~cm}^{-2}$ (Carey et al. 2000).

G79.3P1 shows $\mathrm{CH}_{3} \mathrm{OH}$ emission only from the lowest energy levels in each band. The $\mathrm{CH}_{3} \mathrm{OH}$ spectrum towards G19.30P1 is instead characterized by lines from levels higher in energy, $\left(2_{1} \rightarrow 1_{1}-E ; 6_{0} \rightarrow 6_{-1}\right.$ and $5_{0} \rightarrow 5_{-1}-E ; 5_{1} \rightarrow 4_{1}$, $5_{-2} \rightarrow 4_{-2}$ and $5_{2} \rightarrow 4_{2}-E$ ), which suggest a denser medium.
Moreover the ground state lines $(k=0$ for $A$ state and $k=-1$ for $E$ ) show Gaussian-like profiles with red and blueshifted wings.

G79.3P1 is, thus, an example of a simple source, to be fitted by a single component, while the more complex G19.30P1 region, needs at least a second component to fit the non-Gaussian profiles. As input values, we used typical IRDC values, $T=20 \mathrm{~K}$ and $n\left(\mathrm{H}_{2}\right)=10^{5} \mathrm{~cm}^{-3} \cdot N\left(\mathrm{CH}_{3} \mathrm{OH}\right)$ for $A$ - and $E$-states have been chosen equal to $10^{14} \mathrm{~cm}^{-2}$ using $N\left(\mathrm{H}_{2}\right)=10^{23} \mathrm{~cm}^{-2}$ and assuming $\left[\mathrm{CH}_{3} \mathrm{OH} / \mathrm{H}_{2}\right]=10^{-9}$. The sizes of the $\mathrm{CH}_{3} \mathrm{OH}$ regions are based on BIMA maps of $\mathrm{CH}_{3} \mathrm{OH}$ at $3 \mathrm{~mm}$ (Wyrowski 2004), 32" for G79.3P1 and 12" for G19.30P1. Figures 10a-c show the fitted spectra overlaid on the real data toward G19.30P1.

Table 1 lists the best fit results and the $1 \sigma$ fit range for our estimates of $T_{K}, n\left(\mathrm{H}_{2}\right), N\left(\mathrm{CH}_{3} \mathrm{OH}\right)-A$ and $E$ towards the two sources; for both sources, the fit gives temperatures higher that what found by (Pillai et al. 2004) from $\mathrm{NH}_{3}(2,2),(1,1)$, which trace only the cooler, extended envelope, thus resulting in underestimate of $T_{K}$. Besides the satisfactory agreement between data and fit $\left(\chi_{v_{\mathrm{d}}}=3\right.$ for G19.30P1, $\chi_{v_{\mathrm{d}}}=0.2$ for G79.3P1), some lines, mostly the $2_{k} \rightarrow 1_{k}$ band, are underestimated in intensities. This behaviour is more marked for G19.30P1, for which the $\chi^{2}$ analysis does not allow a reliable estimate of temperature due to the shallow property of the minimum. The source also shows a more complex structure than G79.3P1. Therefore, we attribute these discrepancies mainly to the fact that real sources are more complex than our model can represent, with a finite number of non-interacting components, thus ignoring alignment, self-absorption, a gradual gradient of the parameters within the source.

G79.3P1 offers, instead, a better agreement of the fit with the data, probably due to a less complex structure. All the lines are well fitted by our model, even the $2_{-1} \rightarrow 1_{-1}-E$ which shows an unusual excitation.

Figure 8 shows the $\chi^{2}$ distribution for G79.3P1 in the $\left(T_{\text {kin }}, n\left(\mathrm{H}_{2}\right)\right)$ plane at the column densities derived for $A$ - and $E$-species; 1, 2 and $3 \sigma$ confidence contours, computed from the 
Table 2. Line parameters.

\begin{tabular}{|c|c|c|c|c|c|c|c|}
\hline \multirow[t]{3}{*}{ Transition } & \multirow{2}{*}{$\begin{array}{c}v \\
(\mathrm{GHz}) \\
\end{array}$} & \multirow{2}{*}{\multicolumn{2}{|c|}{$\tau$}} & \multicolumn{2}{|c|}{$\bar{T} T_{\mathrm{ex}}$} & \multirow[t]{2}{*}{$\overline{\bar{\tau}}$} & \multirow{2}{*}{$\begin{array}{l}T_{\mathrm{ex}} \\
(\mathrm{K})\end{array}$} \\
\hline & & & & $(\mathrm{K})$ & $(\mathrm{K})$ & & \\
\hline & & Core & Outflow & Core & Outflow & & \\
\hline G19.30P1 & & & & & & G79.3P11 & \\
\hline $2_{-1} \rightarrow 1_{-1}-E$ & 96.739 & $-8.8 \times 10^{-1}$ & $-7.2 \times 10^{-2}$ & -13.2 & -8.0 & $-5.8 \times 10^{-1}$ & -162.0 \\
\hline $2_{0} \rightarrow 1_{0}-A$ & 96.741 & 1.0 & $4.7 \times 10^{-1}$ & 34.3 & 20.3 & $1.7 \times 10^{-1}$ & 13.0 \\
\hline $2_{0} \rightarrow 1_{0}-E$ & 96.744 & $5.2 \times 10^{-1}$ & $2.3 \times 10^{-1}$ & 16.3 & 7.9 & $3.7 \times 10^{-2}$ & 8.4 \\
\hline $2_{1} \rightarrow 1_{1}-E$ & 96.755 & $7.2 \times 10^{-2}$ & $2.3 \times 10^{-2}$ & 34.7 & 9.4 & & \\
\hline $6_{0} \rightarrow 6_{-1}-E$ & 157.048 & 3.1 & $2.8 \times 10^{-1}$ & 7.0 & 2.9 & & \\
\hline $5_{0} \rightarrow 5_{-1}-E$ & 157.179 & 3.9 & $8.1 \times 10^{-1}$ & 7.4 & 2.9 & $5.2 \times 10^{-2}$ & 9.6 \\
\hline $4_{0} \rightarrow 4_{-1}-E$ & 157.246 & 4.3 & 2.1 & 7.7 & 3.1 & $1.5 \times 10^{-1}$ & 3.3 \\
\hline $1_{0} \rightarrow 1_{-1}-E$ & 157.270 & $6.2 \times 10^{-1}$ & $9.7 \times 10^{-1}$ & 26.0 & 6.5 & $1.8 \times 10^{-1}$ & 6.8 \\
\hline $3_{0} \rightarrow 3_{-1}-E$ & 157.272 & 4.1 & 1.6 & 8.0 & 3.6 & $2.8 \times 10^{-1}$ & 3.8 \\
\hline $2_{0} \rightarrow 2_{-1}-E$ & 157.276 & 3.5 & 2.1 & 8.1 & 4.2 & $3.7 \times 10^{-1}$ & 4.5 \\
\hline $5_{0} \rightarrow 4_{0}-E$ & 241.700 & $6.8 \times 10^{-1}$ & $9.7 \times 10^{-2}$ & 37.4 & 11.5 & $1.4 \times 10^{-2}$ & 9.9 \\
\hline $5_{-1} \rightarrow 4_{-1}-E$ & 241.767 & 1.6 & $9.4 \times 10^{-1}$ & 41.3 & 13.7 & $1.2 \times 10^{-1}$ & 11.3 \\
\hline $5_{0} \rightarrow 4_{0}-A$ & 241.791 & 2.8 & $9.7 \times 10^{-1}$ & 42.4 & 13.8 & $1.7 \times 10^{-1}$ & 11.4 \\
\hline $5_{4} \rightarrow 4_{4}-A$ & 241.806 & $7.6 \times 10^{-3}$ & $3.4 \times 10^{-6}$ & 10.2 & 8.1 & & \\
\hline $5_{-4} \rightarrow 4_{-4}-A$ & 241.806 & $8.5 \times 10^{-3}$ & $3.7 \times 10^{-6}$ & 9.4 & 7.6 & & \\
\hline $5_{-4} \rightarrow 4_{-4}-E$ & 241.813 & $8.6 \times 10^{-4}$ & $1.8 \times 10^{-6}$ & 12.6 & 10.5 & & \\
\hline $5_{4} \rightarrow 4_{4}-E$ & 241.829 & $1.8 \times 10^{-4}$ & $9.7 \times 10^{-7}$ & 17.2 & 12.6 & & \\
\hline $5_{3} \rightarrow 4_{3}-A$ & 241.832 & $1.3 \times 10^{-1}$ & $7.8 \times 10^{-4}$ & 19.8 & 13.2 & & \\
\hline $5_{3} \rightarrow 4_{3}-A$ & 241.833 & $2.9 \times 10^{-2}$ & $6.4 \times 10^{-4}$ & 11.6 & 17.8 & & \\
\hline $5_{2} \rightarrow 4_{2}-A$ & 241.842 & $3.3 \times 10^{-1}$ & $4.1 \times 10^{-4}$ & 17.6 & 12.6 & & \\
\hline $5_{3} \rightarrow 4_{3}-E$ & 241.843 & $1.6 \times 10^{-2}$ & $1.2 \times 10^{-4}$ & 17.2 & 12.1 & & \\
\hline $5_{-3} \rightarrow 4_{-3}-E$ & 241.852 & $2.8 \times 10^{-3}$ & $2.6 \times 10^{-5}$ & 19.4 & 14.2 & & \\
\hline $5_{1} \rightarrow 4_{1}-E$ & 241.879 & $3.1 \times 10^{-1}$ & $1.3 \times 10^{-2}$ & 30.7 & 10.6 & & \\
\hline $5_{2} \rightarrow 4_{2}-A$ & 241.887 & $2.2 \times 10^{-1}$ & $3.4 \times 10^{-4}$ & 24.0 & 14.7 & & \\
\hline $5_{-2} \rightarrow 4_{-2}-E$ & 241.902 & $1.3 \times 10^{-1}$ & $2.1 \times 10^{-2}$ & 23.3 & 10.7 & & \\
\hline $5_{2} \rightarrow 4_{2}-E$ & 241.904 & $3.9 \times 10^{-1}$ & $1.9 \times 10^{-3}$ & 28.4 & 9.3 & & \\
\hline
\end{tabular}

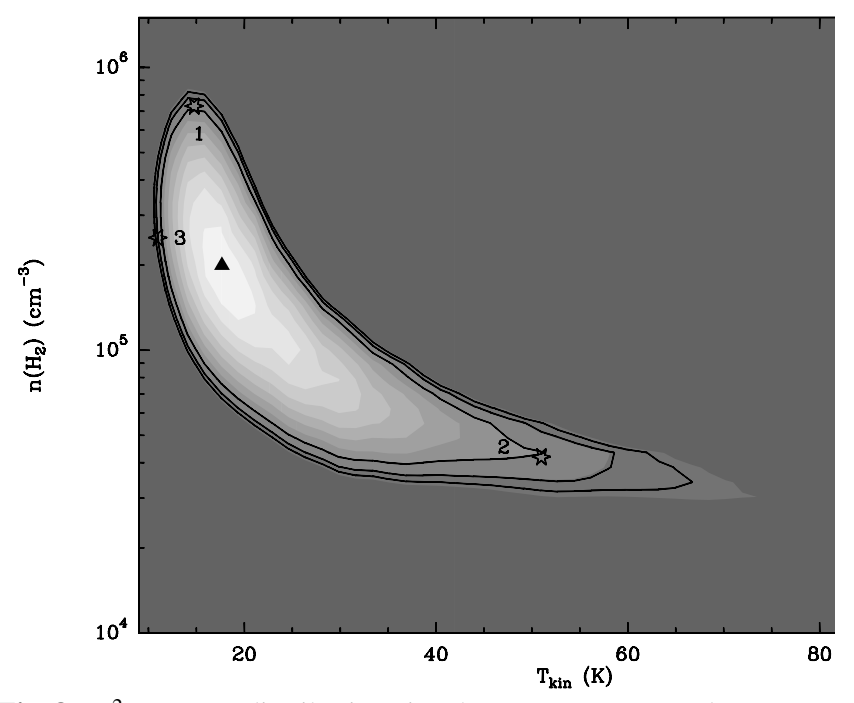

Fig. 8. $\chi^{2}$ square distribution in the $\left[T_{\text {kin }}, n\left(\mathrm{H}_{2}\right)\right]$ plane toward G79.3P1. A black triangle marks the minimum position, $T_{\text {kin }}=17 \mathrm{~K}$, $n\left(\mathrm{H}_{2}\right)=2.5 \times 10^{5} \mathrm{~cm}^{-3}$; stars mark the positions at which spectra of Fig. 11 are taken, which are also labelled by numbers. Overlaid in black the $3 \sigma, 2 \sigma$ and $1 \sigma$ confidence contours. incomplete gamma function (see Press et al. 1992), are shown. Black stars mark the positions within the $1 \sigma$ confidence contour for which spectra are shown in Fig. 11 along with the best fit; even if the fits within the $1 \sigma$ range are still pretty good, major deviations from the observations, mainly at $3 \mathrm{~mm}$ and $2 \mathrm{~mm}$, occur. Since the fit solution falls in the temperature range in which both the $2_{k} \rightarrow 1_{k}$ and the $5_{k} \rightarrow 4_{k}$ bands show a dependence on density and temperature, for G79.3P1 the estimates of $T_{K}$ and $n\left(\mathrm{H}_{2}\right)$ and their uncertainties correlate. This is not true at higher temperature, as in G19.30P1, where the bands have a pure dependence on density. In the case of G79.3P1 we have also compared our LVG results with a similar analysis under the LTE assumption. The agreement between data and LTE fit is usually less satisfactory than in the LVG case, with $\chi_{v_{\mathrm{d}}}=7$, particularly in the $J_{0} \rightarrow J_{-1}-E$ band at $157 \mathrm{GHz}$, which is a known Class II maser series of lines, where the predicted spectrum differs significantly from the measured data. The best fit under the LTE approximation is found at $T=10 \mathrm{~K}$ and for similar values for column densities. The LTE fit overlaid on the data is shown in Fig. 9. 

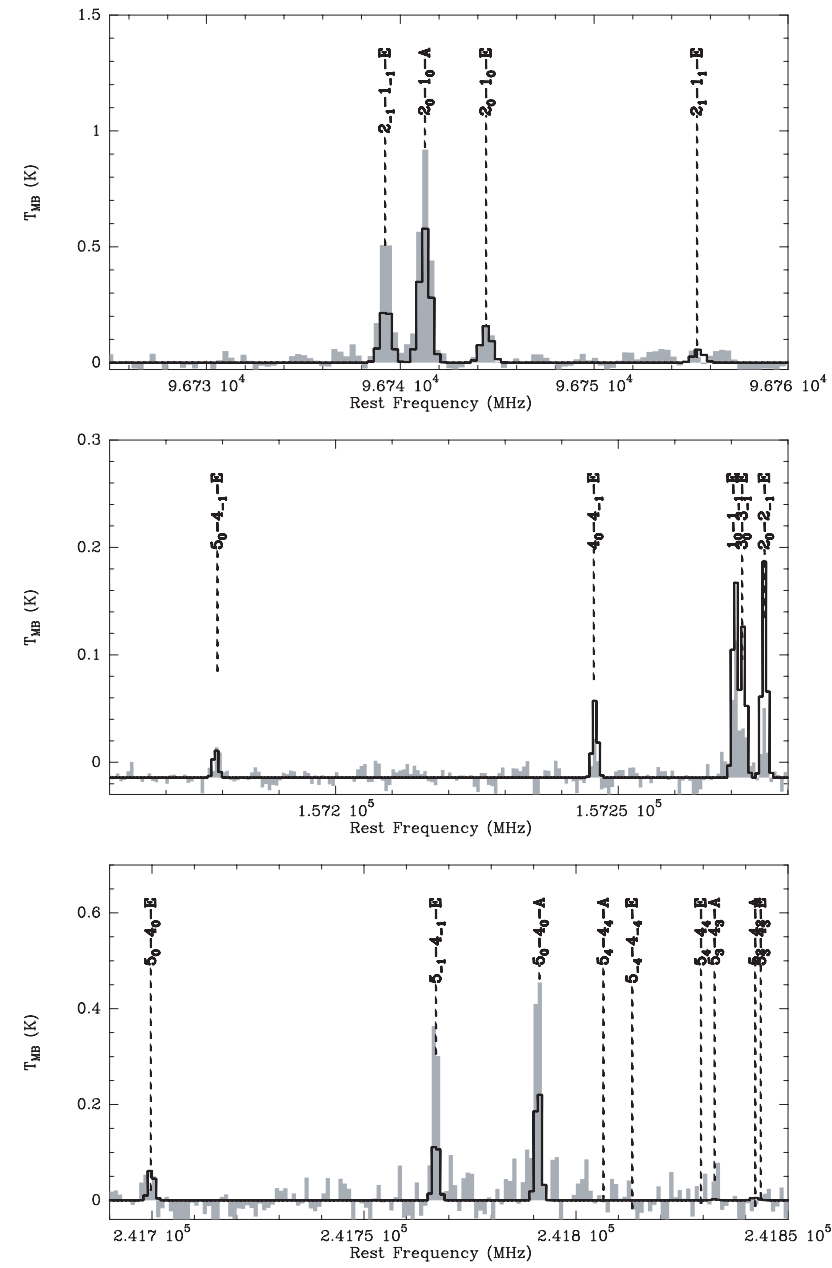

(a)
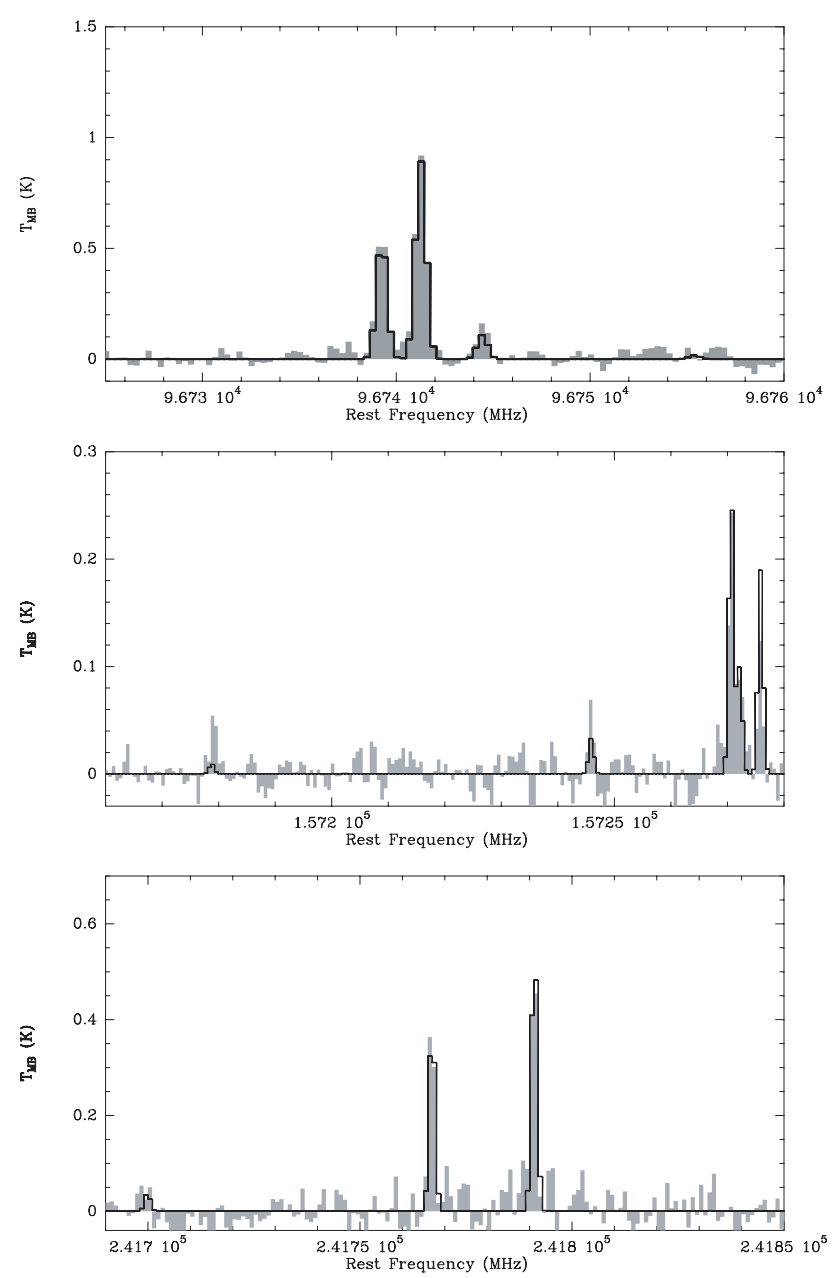

(b)

Fig. 9. a) Best LTE fit, $\left(T_{\text {kin }}=10 \mathrm{~K}, N\left(\mathrm{CH}_{3} \mathrm{OH}-A\right)=7 \times 10^{13} \mathrm{~cm}^{-2}, N\left(\mathrm{CH}_{3} \mathrm{OH}-E\right)=4.6 \times 10^{13} \mathrm{~cm}^{-2}\right)$ toward G79.3P1 (J2000 coordinates: $\mathrm{RA}=20^{\mathrm{h}} 32^{\mathrm{m}} 21^{\prime \prime} 8$, Dec $\left.=40^{\circ} 20^{\prime} 08^{\prime \prime}\right)$ overlaid on the real data. For comparison the best LVG fit $\left(T_{\text {kin }}=17 \mathrm{~K}, n\left(\mathrm{H}_{2}\right)=2.5 \times 10^{5} \mathrm{~cm}^{-3}\right.$, $\left.N\left(\mathrm{CH}_{3} \mathrm{OH}-\mathrm{A}\right)=7.9 \times 10^{13} \mathrm{~cm}^{-2}, N\left(\mathrm{CH}_{3} \mathrm{OH}-E\right)=5.7 \times 10^{13} \mathrm{~cm}^{-2}\right)$ is shown in b).

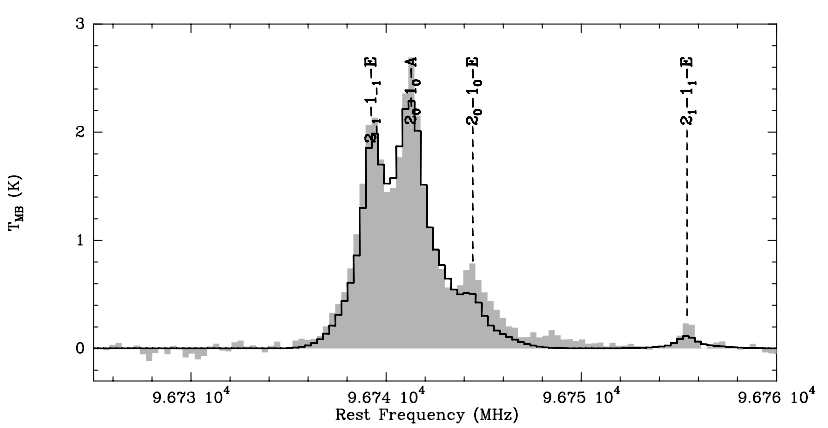

(a)

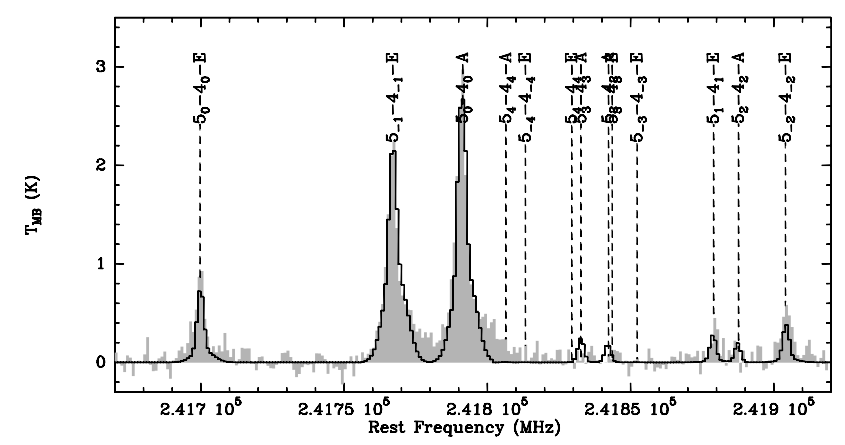

(c)

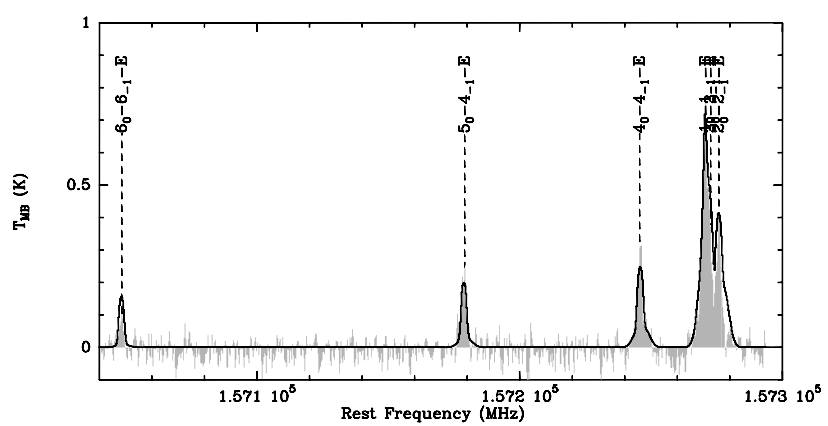

(b)

Fig. 10. Spectra toward G19.30P1 (J2000 coordinates: RA = $18^{\mathrm{h}} 25^{\mathrm{m}} 58^{\prime \prime} 5$, Dec $\left.=-12^{\circ} 03^{\prime} 59^{\prime \prime}\right)$, taken with the IRAM $30 \mathrm{~m}$ telescope. The $2 \mathrm{~mm}$ and $1 \mathrm{~mm}$ data are smoothed for the difference in the beam size with the $3 \mathrm{~mm}$ data. Overlaid in black the synthetic spectra resulting from the fit. 

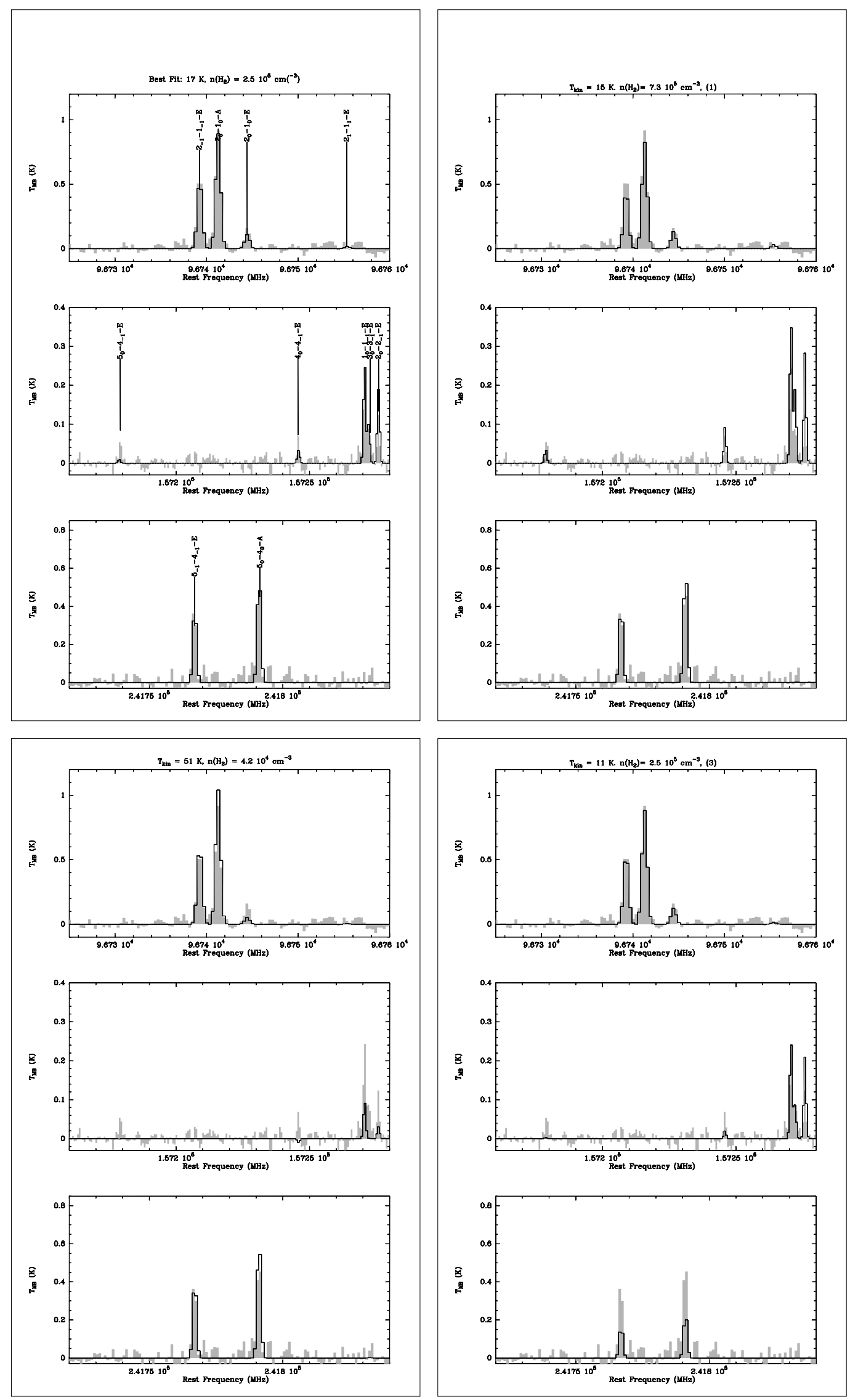

Fig. 11. Synthetic spectra corresponding to different positions within the $1 \sigma$ confidence surface overlaid on the spectra observed toward G79.3P1. For comparison, the best fit spectrum is also shown. Major deviations in the $3 \mathrm{~mm}$ and $2 \mathrm{~mm}$ lines occur in the fits at the $1 \sigma$ level. 


\section{Conclusions}

Using a spherical LVG model to describe the molecular excitation, we have carried out a detailed analysis of the tracing properties of $\mathrm{CH}_{3} \mathrm{OH}$ in the millimeter and submillimeter spectrum. We found that many millimeter line ratios are mainly sensitive to spatial density in the range $10^{5}-10^{8} \mathrm{~cm}^{-3}$, while in the submillimeter range a strong dependence on kinetic temperature up to $150 \mathrm{~K}$ is found. These two results together strongly suggest the use of $\mathrm{CH}_{3} \mathrm{OH}$ as tracer of physical conditions in low-, intermediate, and high-mass star forming regions.

We have also presented an innovative way of analysing $\mathrm{CH}_{3} \mathrm{OH}$ spectra which is based on a simultaneous fitting of all the lines observed towards a given position. The application of this technique to two IRDCs, G19.30P1 and G79.3P1, has been discussed.

Acknowledgements. We would like to take this opportunity to thank the IRAM staff for their support to this project. We are also grateful to M. Walmsley and W. Batrla for valuable discussions and to the anonymous referee for helpful comments.

\section{References}

Bachiller, R., Codella, C., Colomer, F., Liechti, S., \& Walmsley, C. M. 1998, A\&A, 335, 266

Beuther, H., Schilke, P., Menten, K. M., et al. 2002, ApJ, 566, 945

Carey, S. J., Clark, F. O., Egan, M. P., et al. 1998, ApJ, 508, 721

Carey, S. J., Feldman, P. A., Redman, R. O., et al. 2000, ApJ, 543, L157

Cesaroni, R., \& Walmsley, C. M. 1991, A\&A, 241, 537

Comito, C., Schilke, P., Phillips, T., et al. 2004, submitted

Danby, G., Flower, D. R., Valiron, P., Schilke, P., \& Walmsley, C. M. 1988, MNRAS, 235, 229

de Jong, T., Dalgarno, A., \& Chu, S.-I. 1975, ApJ, 199, 69

Dickens, J. E., Irvine, W. M., Snell, R. L., et al. 2000, ApJ, 542, 870

Egan, M. P., Shipman, R. F., Price, S. D., et al. 1998, ApJ, 494, L199

Friberg, P., Hjalmarson, A., Madden, S. C., \& Irvine, W. M. 1988, A\&A, 195, 281

Hogerheijde, M. R., \& van der Tak, F. F. S. 2000, A\&A, 362, 697
Kalenskii, S. V., Dzura, A. M., Booth, R. S., Winnberg, A., \& Alakoz, A. V. 1997, A\&A, 321, 311

Lees, R. M., \& Baker, J. 1968, J. Chem. Phys., 48, 5299

Lees, R. M., \& Haque, S. 1974, Canadian J. Phys., 52, 2250

Müller, H. S. P., Thorwirth, S., Roth, D. A., \& Winnewisser, G. 2001, A\&A, 370, L49

Mangum, J. G., \& Wootten, A. 1993, ApJS, 89, 123

Mekhtiev, M. A., Godfrey, P. D., \& Hougen, J. T. 1999, J. Mol. Spectrosc., 194, 171

Menten, K. M., Walmsley, C. M., Henkel, C., \& Wilson, T. L. 1986, A\&A, 157, 318

Menten, K. M., Walmsley, C. M., Henkel, C., \& Wilson, T. L. 1988, A\&A, 198, 253

Mundy, L. G., Evans, N. J., Snell, R. L., \& Goldsmith, P. F. 1987, ApJ, 318,392

Olmi, L., Cesaroni, R., \& Walmsley, C. M. 1993, A\&A, 276, 489

Pillai, T., Wyrowski, F., Carey, S., \& Egan, M. 2004, in preparation

Pottage, J. T., Flower, D. R., \& Davis, S. L. 2001, J. Phys. B: At. Mol. Phys., 34, 3313

Pottage, J. T., Flower, D. R., \& Davis, S. L. 2002, J. Phys. B: At. Mol. Phys., 35, 2541

Press, W., Teukolsky, S., Vetterling, W., \& Flannery, B. 1992, Numerical Recipes in Fortran, The Art of Scientific Computing (Cambridge University Press)

Ray, B. 1932, Zeitschrift für Physik, 78, 74

Sastry, K. V. L. N., Lees, R. M., \& Van der Linde, J. 1981, J. Mol. Spectrosc., 88, 229

Schilke, P., Phillips, T. G., \& Mehringer, D. M. 1999, in The Physics and Chemistry of the Interstellar Medium, Proceedings of the 3rd Cologne-Zermatt Symposium, 1998, ed. V. Ossenkopf, J. Stutzki, \& G. Winnewisser, GCA-Verlag Herdecke, 330

Snell, R. L., Goldsmith, P. F., Erickson, N. R., Mundy, L. G., \& Evans, N. J. 1984, ApJ, 276, 625

Swade, D. 1989, ApJ, 345, 828

Tafalla, M., Myers, P. C., Caselli, P., Walmsley, C. M., \& Comito, C. 2002, ApJ, 569, 815

Townes, C., \& Schawlow, A. 1996, Microwave Spectroscopy (New York: Dover Pub.)

Walmsley, C. M., Menten, K. M., Batrla, W., \& Matthews, H. E. 1988, A\&A, 197, 271

Walmsley, C. M., \& Ungerechts, H. 1983, A\&A, 122, 164

Wyrowski, F. 2004, in preparation

Xu, L.-H., \& Hougen, J. T. 1995, J. Mol. Spectrosc., 137, 540

Xu, L.-H., \& Lovas, F. L. 1997, J. Phys. Chem. Ref. Data, 26, 17 